\title{
AN INVERSE RANDOM SOURCE PROBLEM FOR THE TIME FRACTIONAL DIFFUSION EQUATION DRIVEN BY A FRACTIONAL BROWNIAN MOTION
}

\author{
XIAOLI FENG, PEIJUN LI, AND XU WANG
}

\begin{abstract}
This paper is concerned with the mathematical analysis of the inverse random source problem for the time fractional diffusion equation, where the source is assumed to be driven by a fractional Brownian motion. Given the random source, the direct problem is to study the stochastic time fractional diffusion equation. The inverse problem is to determine the statistical properties of the source from the expectation and variance of the final time data. For the direct problem, we show that it is well-posed and has a unique mild solution under a certain condition. For the inverse problem, the uniqueness is proved and the instability is characterized. The major ingredients of the analysis are based on the properties of the Mittag-Leffler function and the stochastic integrals associated with the fractional Brownian motion.
\end{abstract}

\section{INTRODUCTION}

In the last two decades, the fractional derivative equations (FDEs) have received ever-increasing attention by many researchers due to their potential applications in modeling real physical phenomena. For examples, the FDE can be used to describe the anomalous diffusion in a highly heterogeneous aquifer [1], the relaxation phenomena in complex viscoelastic materials [10], the anomalous diffusion in an underground environmental problem [13], and a non-Markovian diffusion process with memory [26]. We refer to [11] for some recent advances in theory and simulation of the fractional diffusion processes.

Motivated by significant scientific and industrial applications, the field of inverse problems has undergone a tremendous growth in the last several decades since Calderón proposed an inverse conductivity problem. Recently, the inverse problems on FDEs have also progressed into an area of intense research activity. In particular, for the time or time-space fractional diffusion equations, the inverse source problems have been widely investigated mathematically and numerically. Compared with the semilinear problem [25], many more results are available for the linear problems. The linear inverse source problems for fractional diffusion equations can be broadly classified into the following six cases: (1) determining a space-dependent source term from the space-dependent data [3, 9, 18, 19, 34, 36, 38, 40, 42, 45, 46]; (2) determining a time-dependent source term from the timedependent data [14, 23, 24, 33, 43]; (3) determining a time-dependent source term from the spacedependent data 2,15]; (4) determining a space-dependent source term from the time-dependent data [47; (5) determining a space-dependent source term from the boundary data [41; (6) determining a general source from the time-dependent data [27]. Despite a considerable amount of work done so far, the rigorous mathematical theory is still lacking [16], especially for the inverse problems where the sources contain uncertainties, which are known as the inverse random source problems.

The inverse random source problems belong to a category of stochastic inverse problems, which refer to inverse problems that involve uncertainties. Compared to deterministic inverse problems, stochastic inverse problems have substantially more difficulties on top of the existing obstacles due to the randomness and uncertainties. There are some work done for the inverse random source

2010 Mathematics Subject Classification. 35R30, 35R60, 65M32.

Key words and phrases. fractional diffusion equation, inverse source problem, fractional Brownian motion, uniqueness, ill-posedness.

The research is supported in by part the NSF grant DMS-1912704. 
scattering problems, where the wave propagation is governed by the stochastic Helmholtz equation driven by the white noise. In [8], it was shown that the correlation of the random source could be determined uniquely by the correlation of the random wave field. Recently, an effective computational model was developed in [4, 7, 20 22, the goal was to reconstruct the statistical properties of the random source such as the mean and variance from the boundary measurement of the radiated random wave field at multiple frequencies.

The work is very rare for the inverse random source problems of the fractional diffusion equations. In [29], the authors presented a study on the random source problem for the fractional diffusion equation. Specifically, they considered the following initial-boundary value problem:

$$
\begin{cases}\partial_{t}^{\alpha} u(x, t)-\Delta u(x, t)=f(x) h(t)+g(x) \dot{W}(t), & (x, t) \in D \times(0, T), \\ u(x, t)=0, & (x, t) \in \partial D \times[0, T], \\ u(x, 0)=0, & x \in \bar{D},\end{cases}
$$

where $D$ is a bounded domain with the Lipschitz boundary $\partial D, f$ and $g$ are deterministic functions with compact supports contained in $D, h$ is also a deterministic function, $W$ and $\dot{W}$ are the Brownian motion and the white noise, respectively, and $\partial_{t}^{\alpha} u(x, t), 0<\alpha \leq 1$ is the Caputo fractional derivative given by

$$
\partial_{t}^{\alpha} u(x, t)= \begin{cases}\frac{1}{\Gamma(1-\alpha)} \int_{0}^{t} \frac{\partial u(x, s)}{\partial s} \frac{d s}{(t-s)^{\alpha}}, & 0<\alpha<1, \\ \partial_{t} u(t, x), & \alpha=1 .\end{cases}
$$

Here $\Gamma(\alpha)=\int_{0}^{\infty} e^{-s} s^{\alpha-1} d s$ is the Gamma function. For the model problem (1.1), the authors studied the inverse problem of reconstructing $f(x)$ and $|g(x)|$ from the statistics of the final time data $u(x, T)$ with $\frac{1}{2}<\alpha<1$. It was shown that $f$ and $|g|$ can be uniquely determined by the expectation and covariance of the final data, respectively. Besides, they also showed that the inverse problem is not stable in the sense that a small variance of the data may lead to a huge error of the reconstruction. Naturally, one may ask the following two questions:

Q1. Can the results be extended to $0<\alpha<1$ for the Brownian motion?

Q2. Can the results be extended to the fractional Brownian motion?

Motivated by above reasons, the main purpose of this paper is to study the inverse source problem for the time fractional diffusion equation, where the source is assumed to be driven by a more general stochastic process: the fractional Brownian motion $B^{H}(t)$ with $0<\alpha \leq 1,0<H<1$, where $H$ is called the Hurst index of the fractional Brownian motion. Clearly, the model equation (1.1) is reduced to the classical heat conduction equation with the Brownian motion for $\alpha=1$. In this work, we give confirmative answers to Q1 and Q2. For Q1, due to the singular integral (see Lemma 3.4 in [29] or the proof later in this paper), the results can not be extended; for Q2, the results can be extended as long as $\alpha+H>1$. For the restriction $\alpha+H>1$, it is not difficult to understand since both $H$ and $\alpha$ imply some smoothness requirement of the solution for the model equation.

The rest of this paper is organized as follows. In Section 2, we introduce some preliminaries for the time-fractional diffusion equations and the Mittag-Leffler function. Section 3 is concerned with the well-posedness of the direct problem. Section 4 is devoted to the inverse problem. The two cases $0<H<\frac{1}{2}$ and $\frac{1}{2}<H<1$ are discussed separately for both of the direct and inverse problems. The paper is concluded with some general remarks and directions for future research in Section 5. To make the paper easily accessible, some necessary notations and useful results are provided in Appendix on the fractional Brownian motion.

\section{Preliminaries}

Let the triple $(\Omega, \mathcal{F}, P)$ be a complete probability space on which the fractional Brownian motion $B^{H}$ is defined (see Appendix for the details). Here $\Omega$ is a sample space, $\mathcal{F}$ is a $\sigma$-algebra on $\Omega$, and 
$P$ is a probability measure on the measurable space $(\Omega, \mathcal{F})$. If $X$ is a random variable, $\mathbb{E}(X)$ and $\mathbb{V}(X)=\mathbb{E}(X-\mathbb{E}(X))^{2}=\mathbb{E}\left(X^{2}\right)-(\mathbb{E}(X))^{2}$ are the expectation and variance of $X$, respectively. If $X, Y$ are two random variables, $\operatorname{Cov}(X, Y)=\mathbb{E}[(X-\mathbb{E}(X))(Y-\mathbb{E}(Y))]$ denotes the covariance of $X$ and $Y$.

Consider initial-boundary value problem of the fractional diffusion equation with a random source driven by the fractional Brownian motion

$$
\begin{cases}\partial_{t}^{\alpha} u(x, t)-\Delta u(x, t)=f(x) h(t)+g(x) \dot{B}^{H}(t), & (x, t) \in D \times(0, T), \\ u(x, t)=0, & (x, t) \in \partial D \times[0, T], \\ u(x, 0)=0, & x \in \bar{D}\end{cases}
$$

Let $\left\{\lambda_{k}, \varphi_{k}\right\}_{k=1}^{\infty}$ be the eigensystem of the operator $-\Delta$ with the homogeneous Dirichlet boundary condition in $D$. It is known that the eigenvalues satisfy $0<\lambda_{1} \leq \lambda_{2} \leq \cdots \leq \lambda_{k} \leq \cdots, \lambda_{k} \rightarrow \infty, k \rightarrow$ $\infty$ and the eigen-functions $\left\{\varphi_{k}\right\}_{k=1}^{\infty}$ form a complete and orthogonal basis in $L^{2}(D)$. It follows from the separation of variables that the solution of (2.1) can be written as

$$
u(x, t, \omega)=\sum_{k=1}^{\infty} u_{k}(t, \omega) \varphi_{k}(x),
$$

where $\omega \in \Omega$ and $u_{k}(t, \omega)$ satisfies the stochastic fractional differential equation

$$
\left\{\begin{array}{l}
D_{t}^{\alpha} u_{k}(t, \omega)+\lambda_{k} u_{k}(t, \omega)=f_{k} h(t)+g_{k} \dot{B}^{H}(t), \quad t \in(0, T) \\
u_{k}(0)=0
\end{array}\right.
$$

Here $f_{k}=\left(f, \varphi_{k}\right)_{L^{2}(D)}$ and $g_{k}=\left(g, \varphi_{k}\right)_{L^{2}(D)}$. When $g_{k}=0, k \in \mathbb{N}$, the corresponding deterministic fractional differential equation is

$$
\left\{\begin{array}{l}
D_{t}^{\alpha} u_{k}(t)+\lambda_{k} u_{k}(t)=f_{k} h(t), \quad t \in(0, T) \\
u_{k}(0)=0
\end{array}\right.
$$

whose solution can be obtained directly by applying the following Lemma. The proof can be found in [17, Page 230] or [31, Example 4.3].

Lemma 2.1. Consider the Cauchy problem for the fractional differential equation:

$$
\begin{cases}D_{t}^{\alpha} v(t)-\lambda v(t)=f(t), & t \in(0, T), \\ \frac{d^{n} v}{d t^{n}}(0)=v_{n}, & n=0, \ldots,\lfloor\alpha\rfloor .\end{cases}
$$

If $f(t) \in C^{0, \gamma}$ with $0 \leq \gamma \leq \alpha$, then the Cauchy problem (2.3) has a unique solution given by

$$
v(t)=\sum_{n=0}^{\lfloor\alpha\rfloor} v_{n} t^{n} E_{\alpha, n+1}\left(\lambda t^{\alpha}\right)+\int_{0}^{t}(t-\tau)^{\alpha-1} E_{\alpha, \alpha}\left(\lambda(t-\tau)^{\alpha}\right) f(\tau) d \tau,
$$

where $E_{\alpha, \beta}$ is the Mittag-Leffler function (see 2.7)).

By Lemma 2.1, we can obtain a mild solution of (2.2), which gives a mild solution to the initialboundary value problem of the stochastic fractional diffusion equation (1.1). Let us first give some assumptions in order to understand the solution.

Assumption 1. Assume that $f, g \in L^{2}(D)$ such that $g \neq 0$ and $h \in L^{\infty}(0, T)$ is positive and bounded from below, i.e., there exists $c_{h}>0$ such that $h \geq c_{h}$.

Definition 1. A stochastic process $u: D \times[0, T] \rightarrow L^{2}(D)$ defined by

$$
u(x, t, \omega)=\sum_{k=1}^{\infty}\left(I_{k, 1}(t)+I_{k, 2}(t, \omega)\right) \varphi_{k}(x),
$$


is called a mild solution of the initial-boundary value problem of the stochastic fractional diffusion equation (1.1), where

$$
\begin{aligned}
I_{k, 1}(t) & =f_{k} \int_{0}^{t}(t-\tau)^{\alpha-1} E_{\alpha, \alpha}\left(-\lambda_{k}(t-\tau)^{\alpha}\right) h(\tau) d \tau, \\
I_{k, 2}(t, \omega) & =g_{k} \int_{0}^{t}(t-\tau)^{\alpha-1} E_{\alpha, \alpha}\left(-\lambda_{k}(t-\tau)^{\alpha}\right) d B^{H}(\tau) .
\end{aligned}
$$

Since the Mittag-Leffler function is very important for the analysis, let us state some of its properties. The two-parametric Mittag-Leffler function is defined as

$$
E_{\alpha, \beta}(z)=\sum_{k=0}^{\infty} \frac{z^{k}}{\Gamma(k \alpha+\beta)}, \quad z \in \mathbb{C},
$$

where $\alpha, \beta \in \mathbb{R}$. Obviously, $E_{1,1}(z)=e^{z}$. More information about the Mittag-Leffler function can be found in 12 .

Lemma 2.2. [31, Theorem 1.6] If $0<\alpha<2, \beta$ is an arbitrary real number, $\mu$ is such that $\pi \alpha / 2<\mu<\min \{\pi, \pi \alpha\}$, then there exists a positive constant $C$ such that

$$
\left|E_{\alpha, \beta}(z)\right| \leq \frac{C}{1+|z|}, \quad \mu \leq|\arg (z)| \leq \pi, \quad|z| \geq 0 .
$$

Lemma 2.3. [33, Lemma 3.2] For $\lambda>0, \alpha>0$, we have

$$
\frac{d}{d t} E_{\alpha, 1}\left(-\lambda t^{\alpha}\right)=-\lambda t^{\alpha-1} E_{\alpha, \alpha}\left(-\lambda t^{\alpha}\right), \quad t>0 .
$$

Lemma 2.4. For $\lambda, z \in \mathbb{C}$, we have

$$
\frac{d}{d z}\left(z^{\alpha-1} E_{\alpha, \alpha}\left(-\lambda z^{\alpha}\right)\right)=z^{\alpha-2} E_{\alpha, \alpha-1}\left(-\lambda z^{\alpha}\right) .
$$

Proof. By [12, formula (4.3.1)]

$$
\frac{d}{d z}\left(z^{\alpha-1} E_{\alpha, \alpha}\left(z^{\alpha}\right)\right)=z^{\alpha-2} E_{\alpha, \alpha-1}\left(z^{\alpha}\right)
$$

which completes the proof after using the chain rule.

Lemma 2.5. For any $0<s<t, \lambda_{k}>0$, there exists some constant $C$ such that

$$
\left|t^{\alpha-1} E_{\alpha, \alpha}\left(-\lambda_{k} t^{\alpha}\right)-s^{\alpha-1} E_{\alpha, \alpha}\left(-\lambda_{k} s^{\alpha}\right)\right| \leq C \int_{s}^{t} \frac{r^{\alpha-2}}{1+\lambda_{k} r^{\alpha}} d r .
$$

Proof. By Lemmas 2.4 and 2.2, we have

$$
\frac{d}{d r}\left[r^{\alpha-1} E_{\alpha, \alpha}\left(-\lambda_{k} r^{\alpha}\right)\right]=r^{\alpha-2} E_{\alpha, \alpha-1}\left(-\lambda_{k} r^{\alpha}\right)
$$

and

$$
\left|E_{\alpha, \alpha-1}\left(-\lambda_{k} r^{\alpha}\right)\right| \leq \frac{C}{1+\lambda_{k} r^{\alpha}}
$$

A simple calculation yields that

$$
\begin{aligned}
\left|t^{\alpha-1} E_{\alpha, \alpha}\left(-\lambda_{k} t^{\alpha}\right)-s^{\alpha-1} E_{\alpha, \alpha}\left(-\lambda_{k} s^{\alpha}\right)\right| & =\int_{s}^{t} r^{\alpha-2} E_{\alpha, \alpha-1}\left(-\lambda_{k} r^{\alpha}\right) d r \\
& \leq C \int_{s}^{t} \frac{r^{\alpha-2}}{1+\lambda_{k} r^{\alpha}} d r
\end{aligned}
$$

which completes the proof. 
Lemma 2.6. 32] For $x \geq 0,0 \leq \alpha \leq 1$, the function $E_{\alpha, 1}$ is completely monotonic, i.e.,

$$
(-1)^{n} \frac{d^{n} E_{\alpha, 1}(-x)}{d x^{n}} \geq 0, \quad n=0,1,2, \cdots .
$$

By Lemmas 2.3 and 2.6, we have the following property of $E_{\alpha, \alpha}$.

Lemma 2.7. For $0<\alpha \leq 1, x \geq 0$, there holds $E_{\alpha, \alpha}(-x) \geq 0$ and $x^{\alpha-1} E_{\alpha, \alpha}\left(-\lambda x^{\alpha}\right)$ is monotonically decreasing.

\section{THE DIRECT PROBLEM}

In this section, we discuss the well-posedness of the direct problem. We show that the mild solution (2.4) is well-defined for the initial-boundary value problem of the stochastic fractional diffusion equation (1.1).

It is easy to note that the mild solution (2.4) satisfies

$$
\begin{aligned}
\|u(\cdot, t)\|_{L^{2}(D)}^{2} & =\left\|\sum_{k=1}^{\infty}\left(I_{k, 1}(t)+I_{k, 2}(t, \omega)\right) \varphi_{k}(\cdot)\right\|_{L^{2}(D)}^{2} \\
& =\sum_{k=1}^{\infty}\left(I_{k, 1}(t)+I_{k, 2}(t, \omega)\right)^{2} \leq 2\left(\sum_{k=1}^{\infty} I_{k, 1}^{2}(t)+\sum_{k=1}^{\infty} I_{k, 2}^{2}(t, \omega)\right) .
\end{aligned}
$$

Hence,

$$
\begin{aligned}
\mathbb{E}\left[\|u\|_{L^{2}(D \times[0, T])}^{2}\right] & =\mathbb{E}\left[\int_{0}^{T}\|u(\cdot, t)\|_{L^{2}(D)}^{2} d t\right] \\
& \lesssim \mathbb{E}\left[\int_{0}^{T}\left(\sum_{k=1}^{\infty} I_{k, 1}^{2}(t)+\sum_{k=1}^{\infty} I_{k, 2}^{2}(t, \omega)\right) d t\right] \\
& =\int_{0}^{T}\left(\sum_{k=1}^{\infty} I_{k, 1}^{2}(t)\right) d t+\mathbb{E}\left[\int_{0}^{T}\left(\sum_{k=1}^{\infty} I_{k, 2}^{2}(t, \omega)\right) d t\right] \\
& =\sum_{k=1}^{\infty}\left\|I_{k, 1}\right\|_{L^{2}(0, T)}^{2}+\int_{0}^{T}\left(\sum_{k=1}^{\infty} \mathbb{E}\left[I_{k, 2}^{2}(t, \omega)\right]\right) d t \\
& =: S_{1}+S_{2} .
\end{aligned}
$$

Hereinafter $a \lesssim b$ stands for $a \leqslant C b$, where $C>0$ is a constant.

We shall discuss the sums $S_{1}$ and $S_{2}$ separately. First, let us consider the sum $S_{1}$. Set $G_{\alpha, k}(t)=$ $t^{\alpha-1} E_{\alpha, \alpha}\left(-\lambda_{k} t^{\alpha}\right)$. By (2.5), it is easy to see that $I_{k, 1}(t)=f_{k}\left(G_{\alpha, k} * h\right)(t)$. Using the Young convolution inequality yields

$$
\left\|I_{k, 1}\right\|_{L^{2}(0, T)} \leq\left|f_{k}\right|\left\|G_{\alpha, k}\right\|_{L^{1}(0, T)}\|h\|_{L^{2}(0, T)} .
$$

It follows from Lemma 2.2 that

$$
\left\|G_{\alpha, k}\right\|_{L^{1}(0, T)}=\int_{0}^{T}\left|t^{\alpha-1} E_{\alpha, \alpha}\left(-\lambda_{k} t^{\alpha}\right)\right| d t \lesssim \int_{0}^{T} t^{\alpha-1} d t=\frac{T}{\alpha} .
$$

Combining (3.1)-(3.3), we obtain

$$
S_{1} \leq \frac{T^{2}}{\alpha^{2}} \sum_{k=1}^{\infty}\left|f_{k}\right|^{2}\|h\|_{L^{2}(0, T)}^{2} \lesssim\|h\|_{L^{2}(0, T)}^{2}\|f\|_{L^{2}(D)}^{2} .
$$


Next, we estimate the sum $S_{2}$. By (2.6), we know that

$$
\begin{aligned}
\mathbb{E}\left[I_{k, 2}^{2}(t, \omega)\right] & =\mathbb{E}\left[g_{k}^{2}\left(\int_{0}^{t}(t-\tau)^{\alpha-1} E_{\alpha, \alpha}\left(-\lambda_{k}(t-\tau)^{\alpha}\right) d B^{H}(\tau)\right)^{2}\right] \\
& =g_{k}^{2} \mathbb{E}\left[\left(\int_{0}^{t}(t-\tau)^{\alpha-1} E_{\alpha, \alpha}\left(-\lambda_{k}(t-\tau)^{\alpha}\right) d B^{H}(\tau)\right)^{2}\right]
\end{aligned}
$$

The case $H=\frac{1}{2}, \alpha \in\left(\frac{1}{2}, 1\right)$ has been considered in [29]. We investigate more general $\alpha \in(0,1)$, and discuss the cases $H \in\left(0, \frac{1}{2}\right)$ and $H \in\left(\frac{1}{2}, 1\right)$, respectively, since the stochastic integrals are different.

3.1. The case $H \in\left(0, \frac{1}{2}\right)$. It follows from Appendix on the fractional Brownian motion $B^{H}$ that the stochastic integral in (3.5) with respect to $B^{H}$ satisfies

$$
\begin{aligned}
& \mathbb{E}\left|\int_{0}^{t}(t-\tau)^{\alpha-1} E_{\alpha, \alpha}\left(-\lambda_{k}(t-\tau)^{\alpha}\right) d B^{H}(\tau)\right|^{2} \\
= & \int_{0}^{t}\left[K_{H, t}^{*}\left((t-\cdot)^{\alpha-1} E_{\alpha, \alpha}\left(-\lambda_{k}(t-\cdot)^{\alpha}\right)\right)\right]^{2}(\tau) d \tau \\
= & \int_{0}^{t}\left[K_{H}(t, \tau)(t-\tau)^{\alpha-1} E_{\alpha, \alpha}\left(-\lambda_{k}(t-\tau)^{\alpha}\right)\right. \\
& \left.+\int_{\tau}^{t}\left[(t-u)^{\alpha-1} E_{\alpha, \alpha}\left(-\lambda_{k}(t-u)^{\alpha}\right)-(t-\tau)^{\alpha-1} E_{\alpha, \alpha}\left(-\lambda_{k}(t-\tau)^{\alpha}\right)\right] \frac{\partial K_{H}(u, \tau)}{\partial u} d u\right]^{2} d \tau \\
\lesssim & \int_{0}^{t}\left[\left(\frac{t}{\tau}\right)^{H-\frac{1}{2}}(t-\tau)^{\alpha+H-\frac{3}{2}} E_{\alpha, \alpha}\left(-\lambda_{k}(t-\tau)^{\alpha}\right)\right]^{2} d \tau \\
& +\int_{0}^{t} \tau^{1-2 H}\left[\left(\int_{\tau}^{t} u^{H-\frac{3}{2}}(u-\tau)^{H-\frac{1}{2}} d u\right)(t-\tau)^{\alpha-1} E_{\alpha, \alpha}\left(-\lambda_{k}(t-\tau)^{\alpha}\right)\right]^{2} d \tau \\
& +\int_{0}^{t}\left[\int_{\tau}^{t}\left[(t-u)^{\alpha-1} E_{\alpha, \alpha}\left(-\lambda_{k}(t-u)^{\alpha}\right)-(t-\tau)^{\alpha-1} E_{\alpha, \alpha}\left(-\lambda_{k}(t-\tau)^{\alpha}\right)\right] \frac{\partial K_{H}(u, \tau)}{\partial u} d u\right]^{2} d \tau \\
= & : I_{1}(t)+I_{2}(t)+I_{3}(t),
\end{aligned}
$$

where $K_{H}(u, \tau)$ is given by (A.5). Below we estimate $I_{j}(t), j=1,2,3$.

The estimate of $I_{1}(t)$. By Lemma 2.2, there holds

$$
\begin{aligned}
I_{1}(t) & =\int_{0}^{t}\left(\frac{t}{\tau}\right)^{2 H-1}(t-\tau)^{2 \alpha+2 H-3} E_{\alpha, \alpha}^{2}\left(-\lambda_{k}(t-\tau)^{\alpha}\right) d \tau \\
& \lesssim t^{2 H-1} \int_{0}^{t} \tau^{1-2 H}(t-\tau)^{2 \alpha+2 H-3} d \tau \\
& \leq \int_{0}^{t}(t-\tau)^{2 \alpha+2 H-3} d \tau=\frac{t^{2 \alpha+2 H-2}}{2 \alpha+2 H-2}
\end{aligned}
$$

where we have used the conditions $0<H<\frac{1}{2}, \alpha+H>1$ for the singular integral and the mean value theorem for the definite integral. 
The estimate of $I_{2}(t)$. Using Lemma 2.2, we have

$$
\begin{aligned}
I_{2}(t) & =\int_{0}^{t} \tau^{1-2 H}\left(\int_{\tau}^{t} u^{H-\frac{3}{2}}(u-\tau)^{H-\frac{1}{2}} d u\right)^{2}(t-\tau)^{2 \alpha-2} E_{\alpha, \alpha}^{2}\left(-\lambda_{k}(t-\tau)^{\alpha}\right) d \tau \\
& \lesssim \int_{0}^{t} \tau^{1-2 H}\left(\int_{\tau}^{t} u^{H-\frac{3}{2}}(u-\tau)^{H-\frac{1}{2}} d u\right)^{2}(t-\tau)^{2 \alpha-2} d \tau .
\end{aligned}
$$

Since $H>0$, the integral $\int_{\tau}^{t} u^{H-\frac{3}{2}}(u-\tau)^{H-\frac{1}{2}} d u$ is well-defined. Furthermore, we have from the binomial expansion that

$$
\begin{aligned}
& \int_{\tau}^{t} u^{H-\frac{3}{2}}(u-\tau)^{H-\frac{1}{2}} d u=\int_{\tau}^{t} u^{2 H-2}\left(1-\frac{\tau}{u}\right)^{H-\frac{1}{2}} d u \\
= & \int_{\tau}^{t} u^{2 H-2}\left[\sum_{n=0}^{\infty}\left(\begin{array}{c}
H-\frac{1}{2} \\
n
\end{array}\right)\left(-\frac{\tau}{u}\right)^{n}\right] d u \\
= & \sum_{n=0}^{\infty}\left(\begin{array}{c}
H-\frac{1}{2} \\
n
\end{array}\right)(-1)^{n} \tau^{n} \int_{\tau}^{t} u^{2 H-2-n} d u \\
= & \sum_{n=0}^{\infty}\left(\begin{array}{c}
H-\frac{1}{2} \\
n
\end{array}\right)(-1)^{n} \tau^{n} \frac{t^{2 H-1-n}-\tau^{2 H-1-n}}{2 H-1-n} \\
= & t^{2 H-1} \sum_{n=0}^{\infty}\left(\begin{array}{c}
H-\frac{1}{2} \\
n
\end{array}\right) \frac{(-1)^{n}}{2 H-1-n}\left(\frac{\tau}{t}\right)^{n}-\tau^{2 H-1} \sum_{n=0}^{\infty}\left(\begin{array}{c}
H-\frac{1}{2} \\
n
\end{array}\right) \frac{(-1)^{n}}{2 H-1-n} \\
\leq & \left(t^{2 H-1}-\tau^{2 H-1}\right) \sum_{n=0}^{\infty}\left(\begin{array}{c}
H-\frac{1}{2} \\
n
\end{array}\right) \frac{(-1)^{n}}{2 H-1-n} .
\end{aligned}
$$

It is easy to note from the asymptotic expansion for the binomial coefficients that

$$
\sum_{n=0}^{\infty}\left(\begin{array}{c}
H-\frac{1}{2} \\
n
\end{array}\right) \frac{(-1)^{n}}{2 H-1-n}=A<\infty .
$$

Therefore, (3.8) becomes

$$
\begin{aligned}
I_{2}(t) & \lesssim \int_{0}^{t} \tau^{1-2 H}\left(t^{4 H-2}+\tau^{4 H-2}\right)(t-\tau)^{2 \alpha-2} d \tau \\
& =t^{4 H-2} \int_{0}^{t} \tau^{1-2 H}(t-\tau)^{2 \alpha-2} d \tau+\int_{0}^{t} \tau^{2 H-1}(t-\tau)^{2 \alpha-2} d \tau \\
& =t^{4 H-2} t^{1-2 H} \int_{0}^{t}(t-\tau)^{2 \alpha-2} d \tau+\int_{0}^{t} \tau^{2 H-1} t^{2 \alpha-2}\left(1-\frac{\tau}{t}\right)^{2 \alpha-2} d \tau \\
& =\frac{t^{2 H+2 \alpha-2}}{2 \alpha-1}+t^{2 \alpha-2} \int_{0}^{t} \tau^{2 H-1}\left[\sum_{n=0}^{\infty}\left(\begin{array}{c}
2 \alpha-2 \\
n
\end{array}\right)\left(-\frac{\tau}{t}\right)^{n}\right] d \tau \\
& \lesssim t^{2 H+2 \alpha-2}+t^{2 \alpha-2} \sum_{n=0}^{\infty}\left[\left(\begin{array}{c}
2 \alpha-2 \\
n
\end{array}\right)(-1)^{n} t^{-n} \int_{0}^{t} \tau^{2 H-1+n} d \tau\right] \\
& =t^{2 H+2 \alpha-2}+t^{2 H+2 \alpha-2} \sum_{n=0}^{\infty}\left(\begin{array}{c}
2 \alpha-2 \\
n
\end{array}\right) \frac{(-1)^{n}}{2 H+n}
\end{aligned}
$$


where we have used the conditions $0<H<\frac{1}{2}, \frac{1}{2}<\alpha<1$. Since $2 \alpha-2<0$, we have from the asymptotic expansion for the binomial coefficients again that

$$
0<\sum_{n=0}^{\infty}\left(\begin{array}{c}
2 \alpha-2 \\
n
\end{array}\right) \frac{(-1)^{n}}{2 H+n}=B<\infty
$$

Hence

$$
I_{2}(t) \lesssim t^{2 H+2 \alpha-2}
$$

The estimate of $I_{3}(t)$. Based on Lemma 2.5, for $0<\tau<u<t$, there holds

$$
\begin{aligned}
& \left|(t-u)^{\alpha-1} E_{\alpha, \alpha}\left(-\lambda_{k}(t-u)^{\alpha}\right)-(t-\tau)^{\alpha-1} E_{\alpha, \alpha}\left(-\lambda_{k}(t-\tau)^{\alpha}\right)\right| \\
\lesssim & \int_{t-u}^{t-\tau} r^{\alpha-2} d r \lesssim(t-u)^{\alpha-\frac{3}{2}}(u-\tau)^{\frac{1}{2}}
\end{aligned}
$$

where we have used the fact that $x^{\frac{1}{2}}$ is $\frac{1}{2}$-Hölder continuous for $x>0$. A simple calculation yields that

$$
I_{3}(t) \lesssim \int_{0}^{t}\left[\int_{\tau}^{t}(t-u)^{\alpha-\frac{3}{2}}(u-\tau)^{H-1}\left(\frac{u}{\tau}\right)^{H-\frac{1}{2}} d u\right]^{2} d \tau
$$

The above integral is convergent due to the conditions $H>0, \alpha>\frac{1}{2}$. Since $H \in\left(0, \frac{1}{2}\right)$, we have $\left(\frac{u}{\tau}\right)^{H-\frac{1}{2}}<1$ for $0<\tau<u<t$. Hence

$$
\begin{aligned}
I_{3}(t) & \lesssim \int_{0}^{t}\left[\int_{0}^{t-\tau}(t-\tau-r)^{\alpha-\frac{3}{2}} r^{H-1} d r\right]^{2} d \tau \\
& =\int_{0}^{t}\left[(t-\tau)^{\alpha-\frac{3}{2}} \sum_{n=0}^{\infty}\left(\begin{array}{c}
\alpha-\frac{3}{2} \\
n
\end{array}\right)(-1)^{n}(t-\tau)^{-n} \int_{0}^{t-\tau} r^{n+H-1} d r\right]^{2} d \tau \\
& =\left[\sum_{n=0}^{\infty}\left(\begin{array}{c}
\alpha-\frac{3}{2} \\
n
\end{array}\right) \frac{(-1)^{n}}{n+H}\right]^{2} \int_{0}^{t}(t-\tau)^{2 \alpha+2 H-3} d \tau \lesssim t^{2 \alpha+2 H-2}
\end{aligned}
$$

where we have used the condition $\alpha+H>1$.

Combining (3.6) $-(3.7)$ and (3.9) $-(3.10)$, we obtain for $H \in\left(0, \frac{1}{2}\right)$ that

$$
\mathbb{E}\left|\int_{0}^{t}(t-\tau)^{\alpha-1} E_{\alpha, \alpha}\left(-\lambda_{k}(t-\tau)^{\alpha}\right) d B^{H}(\tau)\right|^{2} \lesssim t^{2 \alpha+2 H-2} .
$$

3.2. The case $H \in\left(\frac{1}{2}, 1\right)$. It follows from Appendix again that the stochastic integral in (3.5) with respect to $B^{H}$ satisfies

$$
\begin{aligned}
& \mathbb{E}\left[\left(\int_{0}^{t}(t-\tau)^{\alpha-1} E_{\alpha, \alpha}\left(-\lambda_{k}(t-\tau)^{\alpha}\right) d B^{H}(\tau)\right)^{2}\right] \\
= & \alpha_{H} \int_{0}^{t} \int_{0}^{t}(t-p)^{\alpha-1} E_{\alpha, \alpha}\left(-\lambda_{k}(t-p)^{\alpha}\right)(t-q)^{\alpha-1} E_{\alpha, \alpha}\left(-\lambda_{k}(t-q)^{\alpha}\right)|p-q|^{2 H-2} d p d q
\end{aligned}
$$


By Lemma 2.2, we have

$$
\begin{aligned}
& \mathbb{E}\left[\left(\int_{0}^{t}(t-\tau)^{\alpha-1} E_{\alpha, \alpha}\left(-\lambda_{k}(t-\tau)^{\alpha}\right) d B^{H}(\tau)\right)^{2}\right] \\
\lesssim & \alpha_{H} \int_{0}^{t} \int_{0}^{t}(t-p)^{\alpha-1}(t-q)^{\alpha-1}|p-q|^{2 H-2} d p d q \\
= & \alpha_{H} \int_{0}^{t} \int_{0}^{t}(t-p)^{\alpha-1}(t-q)^{\alpha-1}|(t-q)-(t-p)|^{2 H-2} d p d q .
\end{aligned}
$$

Let $t-p=\tilde{p}, t-q=\tilde{q}$. A simple calculation gives

$$
\begin{aligned}
& \int_{0}^{t} \int_{0}^{t}(t-p)^{\alpha-1}(t-q)^{\alpha-1}|(t-q)-(t-p)|^{2 H-2} d p d q \\
= & \int_{0}^{t} \int_{0}^{t} \tilde{p}^{\alpha-1} \tilde{q}^{\alpha-1}|\tilde{q}-\tilde{p}|^{2 H-2} d \tilde{p} d \tilde{q} \\
= & \int_{0}^{t} \int_{0}^{\tilde{q}} \tilde{p}^{\alpha-1} \tilde{q}^{\alpha-1}|\tilde{q}-\tilde{p}|^{2 H-2} d \tilde{p} d \tilde{q}+\int_{0}^{t} \int_{\tilde{q}}^{t} \tilde{p}^{\alpha-1} \tilde{q}^{\alpha-1}|\tilde{q}-\tilde{p}|^{2 H-2} d \tilde{p} d \tilde{q} \\
= & 2 \int_{0}^{t} \int_{\tilde{q}}^{t} \tilde{p}^{\alpha-1} \tilde{q}^{\alpha-1}(\tilde{p}-\tilde{q})^{2 H-2} d \tilde{p} d \tilde{q} \\
= & 2 \int_{0}^{t} \int_{\tilde{q}}^{t} \tilde{p}^{\alpha+2 H-3} \tilde{q}^{\alpha-1}\left(1-\frac{\tilde{q}}{\tilde{p}}\right)^{2 H-2} d \tilde{p} d \tilde{q} .
\end{aligned}
$$

Since $\left|\frac{\tilde{q}}{\tilde{p}}\right|<1$, we have from the binomial expansion that

$$
\begin{aligned}
& \int_{0}^{t} \int_{\tilde{q}}^{t} \tilde{p}^{\alpha+2 H-3} \tilde{q}^{\alpha-1}\left(1-\frac{\tilde{q}}{\tilde{p}}\right)^{2 H-2} d \tilde{p} d \tilde{q} \\
= & \int_{0}^{t} \int_{\tilde{q}}^{t} \tilde{p}^{\alpha+2 H-3} \tilde{q}^{\alpha-1}\left(\sum_{n=0}^{\infty}\left(\begin{array}{c}
2 H-2 \\
n
\end{array}\right)\left(-\frac{\tilde{q}}{\tilde{p}}\right)^{n}\right) d \tilde{p} d \tilde{q} \\
= & \int_{0}^{t} \tilde{q}^{\alpha-1} \sum_{n=0}^{\infty}\left(\begin{array}{c}
2 H-2 \\
n
\end{array}\right)(-1)^{n} \tilde{q}^{n}\left(\int_{\tilde{q}}^{t} \tilde{p}^{\alpha+2 H-3-n} d \tilde{p}\right) d \tilde{q} .
\end{aligned}
$$

Note that when $n=0, \alpha+2 H-3-n=-1$ is possible, but when $n \geq 1, \alpha+2 H-3-n=-1$ is impossible. Therefore we discuss the above integral in two cases. 
Case I: $2 H-2=-\alpha$. It follows from the straightforward calculations that

$$
\begin{aligned}
& \int_{0}^{t} \tilde{q}^{\alpha-1} \sum_{n=0}^{\infty}\left(\begin{array}{c}
2 H-2 \\
n
\end{array}\right)(-1)^{n} \tilde{q}^{n}\left(\int_{\tilde{q}}^{t} \tilde{p}^{\alpha+2 H-3-n} d \tilde{p}\right) d \tilde{q} \\
= & \int_{0}^{t} \tilde{q}^{\alpha-1} \sum_{n=0}^{\infty}\left(\begin{array}{c}
2 H-2 \\
n
\end{array}\right)(-1)^{n} \tilde{q}^{n}\left(\int_{\tilde{q}}^{t} \tilde{p}^{-1-n} d \tilde{p}\right) d \tilde{q} \\
= & \int_{0}^{t}\left[\tilde{q}^{\alpha-1} \int_{\tilde{q}}^{t} \tilde{p}^{-1} d \tilde{p}+\tilde{q}^{\alpha-1} \sum_{n=1}^{\infty}\left(\begin{array}{c}
2 H-2 \\
n
\end{array}\right)(-1)^{n} \tilde{q}^{n}\left(\int_{\tilde{q}}^{t} \tilde{p}^{-1-n} d \tilde{p}\right)\right] d \tilde{q} \\
= & \int_{0}^{t} \tilde{q}^{\alpha-1}(\ln t-\ln \tilde{q}) d \tilde{q}+\int_{0}^{t} \tilde{q}^{\alpha-1} \sum_{n=1}^{\infty}\left(\begin{array}{c}
2 H-2 \\
n
\end{array}\right)(-1)^{n} \tilde{q}^{n} \frac{\tilde{q}^{-n}-t^{-n}}{n} d \tilde{q} \\
= & \ln t \int_{0}^{t} \tilde{q}^{\alpha-1} d \tilde{q}-\lim _{\epsilon \rightarrow 0+} \int_{\epsilon}^{t} \tilde{q}^{\alpha-1} \ln \tilde{q} d \tilde{q}+\sum_{n=1}^{\infty}\left(\begin{array}{c}
2 H-2 \\
n
\end{array}\right) \frac{(-1)^{n}}{n}\left(\int_{0}^{t} \tilde{q}^{\alpha-1} d \tilde{q}-t^{-n} \int_{0}^{t} \tilde{q}^{\alpha+n-1} d \tilde{q}\right) \\
= & \frac{t^{\alpha}}{\alpha} \ln t-\lim _{\epsilon \rightarrow 0+}\left(\frac{t^{\alpha}}{\alpha} \ln t-\frac{\epsilon^{\alpha}}{\alpha} \ln \epsilon-\frac{t^{\alpha}-\epsilon^{\alpha}}{\alpha^{2}}\right)+\sum_{n=1}^{\infty}\left(\begin{array}{c}
2 H-2 \\
n
\end{array}\right) \frac{(-1)^{n}}{n} \frac{n t^{\alpha}}{\alpha(\alpha+n)} \\
= & \frac{t^{\alpha}}{\alpha} \frac{1}{\alpha}+\frac{t^{\alpha}}{\alpha} \sum_{n=1}^{\infty}\left(\begin{array}{c}
2 H-2 \\
n
\end{array}\right)(-1)^{n} \frac{1}{\alpha+n} \\
= & \frac{t^{\alpha}}{\alpha} \sum_{n=0}^{\infty}\left(\begin{array}{c}
2 H-2 \\
n
\end{array}\right)(-1)^{n} \frac{1}{\alpha+n},
\end{aligned}
$$

where the integration by parts and L'Hôpital's rule are used. Moreover, the condition $\alpha>0$ can guarantee the convergence of the singular integrals.

Case II: $2 H-2 \neq-\alpha$. Similarly, we have from straightforward calculations that

$$
\begin{aligned}
& \int_{0}^{t} \tilde{q}^{\alpha-1} \sum_{n=0}^{\infty}\left(\begin{array}{c}
2 H-2 \\
n
\end{array}\right)(-1)^{n} \tilde{q}^{n}\left(\int_{\tilde{q}}^{t} \tilde{p}^{\alpha+2 H-3-n} d \tilde{p}\right) d \tilde{q} \\
= & \sum_{n=0}^{\infty}\left(\begin{array}{c}
2 H-2 \\
n
\end{array}\right)(-1)^{n} \frac{1}{\alpha+2 H-2-n}\left(t^{\alpha+2 H-2-n} \int_{0}^{t} \tilde{q}^{n+\alpha-1} d \tilde{q}-\int_{0}^{t} \tilde{q}^{2 \alpha+2 H-3} d \tilde{q}\right),
\end{aligned}
$$

where the conditions $\alpha>0$ and $\alpha+H>1$ are needed to ensure the convergence of the singular integrals. Then we have

$$
\begin{aligned}
& \sum_{n=0}^{\infty}\left(\begin{array}{c}
2 H-2 \\
n
\end{array}\right)(-1)^{n} \frac{1}{\alpha+2 H-2-n}\left(t^{\alpha+2 H-2-n} \int_{0}^{t} \tilde{q}^{n+\alpha-1} d \tilde{q}-\int_{0}^{t} \tilde{q}^{2 \alpha+2 H-3} d \tilde{q}\right) . \\
= & \sum_{n=0}^{\infty}\left(\begin{array}{c}
2 H-2 \\
n
\end{array}\right)(-1)^{n} \frac{1}{\alpha+2 H-2-n}\left(t^{\alpha+2 H-2-n} \frac{t^{n+\alpha}}{n+\alpha}-\frac{t^{2 \alpha+2 H-2}}{2 \alpha+2 H-2}\right) \\
= & \frac{t^{2 \alpha+2 H-2}}{2 \alpha+2 H-2} \sum_{n=0}^{\infty}\left(\begin{array}{c}
2 H-2 \\
n
\end{array}\right)(-1)^{n} \frac{1}{\alpha+n} .
\end{aligned}
$$


Combining Case I and Case II, we get

$$
\begin{aligned}
& \int_{0}^{t} \tilde{q}^{\alpha-1} \sum_{n=0}^{\infty}\left(\begin{array}{c}
2 H-2 \\
n
\end{array}\right)(-1)^{n} \tilde{q}^{n}\left(\int_{\tilde{q}}^{t} \tilde{p}^{\alpha+2 H-3-n} d \tilde{p}\right) d \tilde{q} \\
= & \frac{t^{2 \alpha+2 H-2}}{2 \alpha+2 H-2} \sum_{n=0}^{\infty}\left(\begin{array}{c}
2 H-2 \\
n
\end{array}\right)(-1)^{n} \frac{1}{\alpha+n} .
\end{aligned}
$$

It is easy to know from the asymptotic expansion for the binomial coefficients that the above series is convergent. Therefore,

$$
\mathbb{E}\left[\left(\int_{0}^{t}(t-\tau)^{\alpha-1} E_{\alpha, \alpha}\left(-\lambda_{k}(t-\tau)^{\alpha}\right) d B^{H}(\tau)\right)^{2}\right] \lesssim t^{2 \alpha+2 H-2} .
$$

3.3. Estimates of the solution. In this section, we discuss the stability of the solution. From (3.11)-(3.12) and the analysis for $H=\frac{1}{2}$ in [29], for $0<H<1,0<\alpha \leq 1$ and $\alpha+H>1$, there holds

$$
\mathbb{E}\left[\left(\int_{0}^{t}(t-\tau)^{\alpha-1} E_{\alpha, \alpha}\left(-\lambda_{k}(t-\tau)^{\alpha}\right) d B^{H}(\tau)\right)^{2}\right] \lesssim t^{2 \alpha+2 H-2} .
$$

With the help of (3.13), we obtain the stability estimates for the mild solution (2.4).

Theorem 3.1. Let $0<H<1,0<\alpha \leq 1$ and $\alpha+H>1$. Then the stochastic process $u$ given in 2.4) satisfies

$$
\mathbb{E}\left(\|u\|_{L^{2}(D \times[0, T])}^{2}\right) \lesssim\|h\|_{L^{2}(0, T)}^{2}\|f\|_{L^{2}(D)}^{2}+T^{2 \alpha+2 H-1}\|g\|_{L^{2}(D)}^{2} .
$$

Proof. The proof follows easily from (3.1), (3.4), (3.5), (3.13). Especially, one can check it is also true for $\alpha=1$.

Similarly, we also have the following stability results.

Theorem 3.2. Let $0<H<1,0<\alpha \leq 1$ and $\alpha+H>1$. The supremum of the expected norm of the solution satisfies

$$
\sup _{0 \leqslant t \leqslant T} \mathbb{E}\left[\|u(\cdot, t)\|_{L^{2}(D)}^{2}\right] \lesssim\|h\|_{L^{\infty}(0, T)}^{2}\|f\|_{L^{2}(D)}^{2}+T^{2 \alpha+2 H-2}\|g\|_{L^{2}(D)}^{2} .
$$

Moreover, if condition $g \in H^{2}(D)$ is added, there also holds

$$
\sup _{0 \leqslant t \leqslant T} \mathbb{E}\left[\|u(\cdot, t)\|_{H^{2}(D)}^{2}\right] \lesssim\|h\|_{L^{\infty}(0, T)}^{2}\|f\|_{L^{2}(D)}^{2}+T^{2 \alpha+2 H-2}\|g\|_{H^{2}(D)}^{2} .
$$

Proof. The theorem can be proved by following similar arguments for the case $H=\frac{1}{2}, \alpha \in\left(\frac{1}{2}, 1\right)$ in [29, Lemma 3.5]. The details are omitted for brevity.

Although we only show the details for the Laplacian operator in (2.1), the method can be applied to following initial-bound value problem for the stochastic fractional diffusion equation with the fractional Laplacian operator:

$$
\begin{cases}\partial_{t}^{\alpha} u(x, t)+(-\Delta)^{s} u(x, t)=f(x) h(t)+g(x) \dot{B}^{H}(t), & (x, t) \in D \times(0, T), \\ u(x, t)=0, & (x, t) \in \mathbb{R}^{n} \backslash D \times[0, T] \\ u(x, 0)=0, & x \in D\end{cases}
$$

where $0<\alpha \leq 1,0<s<1,0<H<1$, and $\alpha+H>1$. The fractional Laplacian operator $(-\Delta)^{s}$ is defined as follows [28, formula (3.1)]:

$$
(-\Delta)^{s} u(x, t)=C_{n, s} \text { p.v. } \int_{\mathbb{R}^{n}} \frac{u(x, t)-u(y, t)}{|x-y|^{n+2 s}} d y,
$$


where $C_{n, s}$ is a positive constant depending on $n$ and $s$. Using the properties of the eigensystem for the fractional Laplacian operator $(-\Delta)^{s}$ given in [44, Proposition 2.1], one can also use the method of separation of variables to obtain a mild solution. Then all the results are the same except the second result in Theorem 3.2. But it can be easily shown that if $g \in H^{s}(D)$, then there holds

$$
\sup _{0 \leqslant t \leqslant T} \mathbb{E}\left[\|u(\cdot, t)\|_{H^{s}(D)}^{2}\right] \lesssim\|h\|_{L^{\infty}(0, T)}^{2}\|f\|_{L^{2}(D)}^{2}+T^{2 \alpha+2 H-2}\|g\|_{H^{s}(D)}^{2}
$$

The fractional Sobolev space $H^{s}(D)$ can be found in [28] and related references therein.

\section{THE INVERSE PROBLEM}

In this section, we consider the inverse problem of reconstructing $f$ and $|g|$ from the empirical expectation and correlations of the final time data $u(x, T)$. More specifically, the data may be assumed to be given by

$$
u_{k}(T, \omega)=\left(u(T, \cdot, \omega), \varphi_{k}(\cdot)\right)_{L^{2}(D)} .
$$

We shall discuss the uniqueness and the issue of instability, separately.

It follows from (2.4) $-(2.6)$ that we have

$$
\mathbb{E}\left(u_{k}(T, \omega)\right)=f_{k} \int_{0}^{T}(T-\tau)^{\alpha-1} E_{\alpha, \alpha}\left(-\lambda_{k}(T-\tau)^{\alpha}\right) h(\tau) d \tau
$$

and

$$
\mathbb{V}\left(u_{k}(T, \omega)\right)=g_{k}^{2} \mathbb{E}\left|\int_{0}^{T}(T-\tau)^{\alpha-1} E_{\alpha, \alpha}\left(-\lambda_{k}(T-\tau)^{\alpha}\right) d B^{H}(\tau)\right|^{2} .
$$

Moreover, a straightforward calculation yields that

$$
\begin{aligned}
& \operatorname{Cov}\left(u_{k}(T, \omega), u_{l}(T, \omega)\right) \\
&=g_{k} g_{l} \mathbb{E}\left[\left(\int_{0}^{T}(T-\tau)^{\alpha-1} E_{\alpha, \alpha}\left(-\lambda_{k}(T-\tau)^{\alpha}\right) d B^{H}(\tau)\right)\right. \\
&\left.\times\left(\int_{0}^{T}(T-\tau)^{\alpha-1} E_{\alpha, \alpha}\left(-\lambda_{l}(T-\tau)^{\alpha}\right) d B^{H}(\tau)\right)\right] .
\end{aligned}
$$

Lemma 4.1. Suppose Assumption 1 holds. For each fixed $k \in \mathbb{N}$, there exists a constant $C_{1}>0$ such that

$$
\int_{0}^{T}(T-\tau)^{\alpha-1} E_{\alpha, \alpha}\left(-\lambda_{k}(T-\tau)^{\alpha}\right) h(\tau) d \tau \geq C_{1}>0 .
$$

Proof. Letting $\tilde{\tau}=T-\tau$, we have from Lemma 2.7 and Assumption 1 that

$$
\begin{aligned}
& \int_{0}^{T}(T-\tau)^{\alpha-1} E_{\alpha, \alpha}\left(-\lambda_{k}(T-\tau)^{\alpha}\right) h(\tau) d \tau \\
= & \int_{0}^{T} \tilde{\tau}^{\alpha-1} E_{\alpha, \alpha}\left(-\lambda_{k} \tilde{\tau}^{\alpha}\right) h(T-\tilde{\tau}) d \tilde{\tau} \\
\geq & T^{\alpha-1} E_{\alpha, \alpha}\left(-\lambda_{k} T^{\alpha}\right) \int_{0}^{T} h(T-\tilde{\tau}) d \tilde{\tau} \\
\geq & c_{h} T^{\alpha} E_{\alpha, \alpha}\left(-\lambda_{k} T^{\alpha}\right)=: C_{1}>0,
\end{aligned}
$$

which completes the proof. 
Lemma 4.2. Suppose Assumption 1 holds. For each fixed $k, l \in \mathbb{N}$, there exists a constant $C_{2}>0$ such that

$$
\begin{aligned}
& \mathbb{E}\left[\left(\int_{0}^{T}(T-\tau)^{\alpha-1} E_{\alpha, \alpha}\left(-\lambda_{k}(T-\tau)^{\alpha}\right) d B^{H}(\tau)\right)\right. \\
& \left.\quad \times\left(\int_{0}^{T}(T-\tau)^{\alpha-1} E_{\alpha, \alpha}\left(-\lambda_{l}(T-\tau)^{\alpha}\right) d B^{H}(\tau)\right)\right] \geq C_{2}>0 .
\end{aligned}
$$

Proof. Let $\phi_{k}(s)=(T-s)^{\alpha-1} E_{\alpha, \alpha}\left(-\lambda_{k}(T-s)^{\alpha}\right)$ and

$$
I_{k l}=\mathbb{E}\left[\left(\int_{0}^{T} \phi_{k}(\tau) d B^{H}(\tau)\right)\left(\int_{0}^{T} \phi_{l}(\tau) d B^{H}(\tau)\right)\right] .
$$

We consider $I_{k l}$ for $H \in\left(\frac{1}{2}, 1\right)$ and $H \in\left(0, \frac{1}{2}\right)$, separately.

For $H \in\left(\frac{1}{2}, 1\right)$, we have from (A.4) that

$$
\begin{aligned}
I_{k l} & =\alpha_{H} \int_{0}^{T} \int_{0}^{T} \phi_{k}(r) \phi_{l}(u)|r-u|^{2 H-2} d u d r \\
& =\alpha_{H} \int_{0}^{T} \int_{0}^{T}(T-r)^{\alpha-1} E_{\alpha, \alpha}\left(-\lambda_{k}(T-r)^{\alpha}\right)(T-u)^{\alpha-1} E_{\alpha, \alpha}\left(-\lambda_{l}(T-u)^{\alpha}\right)|r-u|^{2 H-2} d u d r .
\end{aligned}
$$

Set $\tilde{r}=T-r, \tilde{u}=T-u$. A simple calculation yields

$$
I_{k l}=\alpha_{H} \int_{0}^{T} \int_{0}^{T} \tilde{r}^{\alpha-1} E_{\alpha, \alpha}\left(-\lambda_{k} \tilde{r}^{\alpha}\right) \tilde{u}^{\alpha-1} E_{\alpha, \alpha}\left(-\lambda_{l} \tilde{u}^{\alpha}\right)|\tilde{u}-\tilde{r}|^{2 H-2} d \tilde{u} d \tilde{r} .
$$

By Lemma 2.7, the function $\tilde{\tau}^{\alpha-1} E_{\alpha, \alpha}\left(-\lambda_{k} \tilde{\tau}^{\alpha}\right)$ is a monotonically decreasing function with respect to $\tilde{\tau}$. Hence

$$
\begin{aligned}
I_{k l} & \geq \alpha_{H} \int_{0}^{T} \int_{0}^{T} T^{\alpha-1} E_{\alpha, \alpha}\left(-\lambda_{k} T^{\alpha}\right) T^{\alpha-1} E_{\alpha, \alpha}\left(-\lambda_{l} T^{\alpha}\right)|\tilde{u}-\tilde{r}|^{2 H-2} d \tilde{u} d \tilde{r} \\
& =\alpha_{H} T^{2 \alpha-2} E_{\alpha, \alpha}\left(-\lambda_{k} T^{\alpha}\right) E_{\alpha, \alpha}\left(-\lambda_{l} T^{\alpha}\right) \int_{0}^{T} \int_{0}^{T}|\tilde{u}-\tilde{r}|^{2 H-2} d \tilde{u} d \tilde{r} \\
& =\frac{\alpha_{H} T^{2(\alpha+H-1)}}{H(2 H-1)} E_{\alpha, \alpha}\left(-\lambda_{k} T^{\alpha}\right) E_{\alpha, \alpha}\left(-\lambda_{l} T^{\alpha}\right)=: C_{2}>0 .
\end{aligned}
$$

For $H \in\left(0, \frac{1}{2}\right)$, by (A.6), we have

$$
I_{k l}=\left\langle K_{H, T}^{*} \phi_{k}, K_{H, T}^{*} \phi_{l}\right\rangle_{L^{2}(0, T)},
$$

where

$$
\begin{gathered}
\left(K_{H, T}^{*} \phi_{k}\right)(s)=K_{H}(T, s) \phi_{k}(s)+\int_{s}^{T}\left(\phi_{k}(u)-\phi_{k}(s)\right) \frac{\partial K_{H}(u, s)}{\partial u} d u, \\
K_{H}(T, s)=c_{H}\left[\left(\frac{T}{s}\right)^{H-\frac{1}{2}}(T-s)^{H-\frac{1}{2}}-\left(H-\frac{1}{2}\right) s^{\frac{1}{2}-H} \int_{s}^{T} u^{H-\frac{3}{2}}(u-s)^{H-\frac{1}{2}} d u\right], \\
\frac{\partial K_{H}(u, s)}{\partial u}=c_{H}\left(\frac{u}{s}\right)^{H-\frac{1}{2}}(u-s)^{H-\frac{3}{2}} .
\end{gathered}
$$


Obviously, $K_{H}(T, s)>0$ since $H \in\left(0, \frac{1}{2}\right), 0<s<T$. It follows from the mean value theorem that

$$
\begin{aligned}
& \int_{s}^{T}\left(\phi_{k}(u)-\phi_{k}(s)\right) \frac{\partial K_{H}(u, s)}{\partial u} d u \\
= & c_{H} \int_{s}^{T}\left(\phi_{k}(u)-\phi_{k}(s)\right)\left(\frac{u}{s}\right)^{H-\frac{1}{2}}(u-s)^{H-\frac{3}{2}} d u \\
= & c_{H} \int_{s}^{T} \phi_{k}^{\prime}\left(u_{k}^{*}\right)\left(\frac{u}{s}\right)^{H-\frac{1}{2}}(u-s)^{H-\frac{1}{2}} d u \quad\left(s<u_{k}^{*}<u<T\right) \\
= & c_{H} \phi_{k}^{\prime}\left(u_{k}^{* *}\right) \int_{s}^{T}\left(\frac{u}{s}\right)^{H-\frac{1}{2}}(u-s)^{H-\frac{1}{2}} d u \quad\left(s<u_{k}^{* *}<T\right) \\
= & M_{H}(s) \phi_{k}^{\prime}\left(u_{k}^{* *}\right),
\end{aligned}
$$

where

$$
M_{H}(s)=c_{H} \int_{s}^{T}\left(\frac{u}{s}\right)^{H-\frac{1}{2}}(u-s)^{H-\frac{1}{2}} d u>0 .
$$

A simple calculation gives that

$$
\begin{aligned}
I_{k l}= & \left\langle K_{H}(T, s) \phi_{k}(s)+M_{H}(s) \phi_{k}^{\prime}\left(u_{k}^{* *}\right), K_{H}(T, s) \varphi_{l}(s)+M_{H}(s) \phi_{l}^{\prime}\left(u_{l}^{* *}\right)\right\rangle_{L^{2}(0, T)} \\
= & \left\langle K_{H}(T, s) \phi_{k}(s), K_{H}(T, s) \phi_{l}(s)\right\rangle_{L^{2}(0, T)}+\left\langle M_{H}(s) \phi_{k}^{\prime}\left(u_{k}^{* *}\right), M_{H}(s) \phi_{l}^{\prime}\left(u_{l}^{* *}\right)\right\rangle_{L^{2}(0, T)} \\
& +\left\langle K_{H}(T, s) \phi_{k}(s), M_{H}(s) \phi_{l}^{\prime}\left(u_{l}^{* *}\right)\right\rangle_{L^{2}(0, T)}+\left\langle M_{H}(s) \phi_{k}^{\prime}\left(u_{k}^{* *}\right), K_{H}(T, s) \phi_{l}(s)\right\rangle_{L^{2}(0, T)} .
\end{aligned}
$$

It follows Lemma 2.7 again that there holds

$$
\begin{aligned}
& \left\langle K_{H}(T, s) \phi_{k}(s), K_{H}(T, s) \phi_{l}(s)\right\rangle_{L^{2}(0, T)} \\
= & \int_{0}^{T} K_{H}^{2}(T, s) \phi_{k}(s) \phi_{l}(s) d s \\
\geq & T^{2 \alpha-2} E_{\alpha, \alpha}\left(-\lambda_{k} T^{\alpha}\right) E_{\alpha, \alpha}\left(-\lambda_{l} T^{\alpha}\right) \int_{0}^{T} K_{H}^{2}(T, s) d s:=\tilde{c}_{1}>0 .
\end{aligned}
$$

Using Lemmas 2.3 and 2.6, and noting $T-s=t$, we obtain that $\phi_{k}(s)>0$ and $\phi_{k}(s)$ is a monotonically increasing function; $\phi_{k}^{\prime}(s)>0$ and $\phi_{k}^{\prime}(s)$ is a monotonically decreasing function, which imply $\phi_{k}(s) \geq \phi_{k}(0)>0$ and $\varphi_{k}^{\prime}(s) \geq \varphi_{k}^{\prime}(T)>0$ for $0<s<T$. Hence

$$
\left\langle M_{H}(s) \phi_{k}^{\prime}\left(u_{k}^{* *}\right), M_{H}(s) \phi_{l}^{\prime}\left(u_{l}^{* *}\right)\right\rangle_{L^{2}(0, T)} \geq \phi_{k}^{\prime}(T) \phi_{l}^{\prime}(T) \int_{0}^{T} M_{H}^{2}(s) d s:=\tilde{c}_{2}>0 .
$$

Similarly, we have

$$
\left\langle K_{H}(T, s) \phi_{k}(s), M_{H}(s) \phi_{l}^{\prime}\left(u_{l}^{* *}\right)\right\rangle_{L^{2}(0, T)} \geq \phi_{k}(0) \phi_{l}^{\prime}(T) \int_{0}^{T} K_{H}(T, s) M_{H}(s) d s:=\tilde{c}_{3}>0
$$

and

$$
\left\langle M_{H}(s) \phi_{k}^{\prime}\left(u_{k}^{* *}\right), K_{H}(T, s) \phi_{l}(s)\right\rangle_{L^{2}(0, T)} \geq \phi_{k}^{\prime}(T) \phi_{l}(0) \int_{0}^{T} K_{H}(T, s) M_{H}(s) d s:=\tilde{c}_{4}>0 .
$$

Combining the above estimates gives

$$
I_{k l} \geq \sum_{j=1}^{4} \tilde{c}_{j}:=C_{2}>0,
$$

which completes the proof.

Combining (4.1)-(4.3) and Lemmas 4.1 and 4.2, we obtain the uniqueness of the inverse problem. 
Theorem 4.3. Suppose Assumption 1 holds. Then the quantities

$$
\left\{\mathbb{E}\left(u_{k}(T, \omega)\right), \operatorname{Cov}\left(u_{k}(T, \omega), u_{l}(T, \omega)\right): k, l \in \mathbb{N}\right\}
$$

can determine the source terms $f$ and $|g|$ uniquely.

Proof. Since $f, g \in L^{2}(D)$, we have

$$
f(x)=\sum_{k=1}^{\infty} f_{k} \varphi_{k}(x), \quad g(x)=\sum_{k=1}^{\infty} g_{k} \varphi_{k}(x)
$$

which gives that

$$
g^{2}(x)=\left(\sum_{k=1}^{\infty} g_{k} \varphi_{k}(x)\right)\left(\sum_{l=1}^{\infty} g_{l} \varphi_{l}(x)\right)=\sum_{k, l \in \mathbb{N}} g_{k} g_{l} \varphi_{k}(x) \varphi_{l}(x) .
$$

By Lemmas 4.1 and 4.2 , the proof is completed by noting (4.1) and (4.3).

Unfortunately, the inverse source problem is unstable. In [29, Lemma 4.4], the authors have explained the instability for $H=\frac{1}{2}, \alpha \in\left(\frac{1}{2}, 1\right)$. Since the formula (4.1) does not involve the Brownian motion, the instability of recovering $f$ is the same. Therefore, we shall only discuss the instability of recovering $|g|$. It suffices to show that it is unstable to recover $g_{k}^{2}$ in (4.3) when $k=l$, i.e., we shall focus on the estimate of (4.2).

First, we choose $t_{*}$ small enough such that

$$
\frac{1}{1+\lambda_{k} r^{\alpha}} \leq \begin{cases}1 & \text { if } r<t_{*} \\ \frac{1}{\lambda_{k} r^{\alpha}} & \text { if } r>t_{*}\end{cases}
$$

Below we discuss the two different cases $H \in\left(0, \frac{1}{2}\right)$ and $H \in\left(\frac{1}{2}, 1\right)$, separately.

4.1. The case $H \in\left(0, \frac{1}{2}\right)$. We consider the estimate (3.6) with $t=T$ and estimate $I_{j}(T), j=1,2,3$.

The estimate $I_{1}(T)$. A simple calculation yields

$$
\begin{aligned}
I_{1}(T) & =\int_{0}^{T}\left[\left(\frac{T}{\tau}\right)^{H-\frac{1}{2}}(T-\tau)^{\alpha+H-\frac{3}{2}} E_{\alpha, \alpha}\left(-\lambda_{k}(T-\tau)^{\alpha}\right)\right]^{2} d \tau \\
& =\left(\int_{0}^{T-t_{*}}+\int_{T-t_{*}}^{T}\right)\left(\left(\frac{T}{\tau}\right)^{2 H-1}(T-\tau)^{2 \alpha+2 H-3} E_{\alpha, \alpha}^{2}\left(-\lambda_{k}(T-\tau)^{\alpha}\right)\right) d \tau .
\end{aligned}
$$

We have from (4.4) that

$$
\begin{aligned}
& \int_{0}^{T-t_{*}}\left(\frac{T}{\tau}\right)^{2 H-1}(T-\tau)^{2 \alpha+2 H-3} E_{\alpha, \alpha}^{2}\left(-\lambda_{k}(T-\tau)^{\alpha}\right) d \tau \\
\leq & \int_{0}^{T-t_{*}}\left(\frac{T}{\tau}\right)^{2 H-1}(T-\tau)^{2 \alpha+2 H-3} \frac{1}{\lambda_{k}^{2}(T-\tau)^{2 \alpha}} d \tau \\
= & \frac{1}{\lambda_{k}^{2}} \int_{0}^{T-t_{*}}\left(\frac{T}{\tau}\right)^{2 H-1}(T-\tau)^{2 H-3} d \tau .
\end{aligned}
$$


The condition $H>0$ is enough to ensure the convergence of the above singular integral. Moreover, it follows from the binomial expansion that we obtain

$$
\begin{aligned}
& \frac{1}{\lambda_{k}^{2}} \int_{0}^{T-t_{*}}\left(\frac{T}{\tau}\right)^{2 H-1}(T-\tau)^{2 H-3} d \tau \\
= & \frac{T^{4 H-4}}{\lambda_{k}^{2}} \int_{0}^{T-t_{*}} \tau^{1-2 H}\left(1-\frac{\tau}{T}\right)^{2 H-3} d \tau \\
= & \frac{T^{4 H-4}}{\lambda_{k}^{2}} \int_{0}^{T-t_{*}} \tau^{1-2 H}\left(\sum_{n=0}^{\infty}\left(\begin{array}{c}
2 H-3 \\
n
\end{array}\right)(-1)^{n}\left(\frac{1}{T}\right)^{n} \tau^{n}\right) d \tau \\
= & \frac{T^{4 H-4}}{\lambda_{k}^{2}} \sum_{n=0}^{\infty}\left(\begin{array}{c}
2 H-3 \\
n
\end{array}\right)(-1)^{n}\left(\frac{1}{T}\right)^{n} \int_{0}^{T-t_{*}} \tau^{n+1-2 H} d \tau \\
= & \frac{T^{4 H-4}}{\lambda_{k}^{2}} \sum_{n=0}^{\infty}\left(\begin{array}{c}
2 H-3 \\
n
\end{array}\right)(-1)^{n}\left(\frac{1}{T}\right)^{n} \frac{\left(T-t_{*}\right)^{n+2-2 H}}{n+2-2 H} \\
= & \frac{T^{4 H-4}}{\lambda_{k}^{2}}\left(T-t_{*}\right)^{2-2 H} \sum_{n=0}^{\infty}\left(\begin{array}{c}
2 H-3 \\
n
\end{array}\right) \frac{(-1)^{n}}{n+2-2 H}\left(\frac{T-t_{*}}{T}\right)^{n} \\
\lesssim & \frac{1}{\lambda_{k}^{2}} T^{4 H-4}\left(T-t_{*}\right)^{2-2 H} \leq \frac{1}{\lambda_{k}^{2}} T^{2 H-2} .
\end{aligned}
$$

On the other hand, by (4.4), there holds

$$
\begin{aligned}
& \int_{T-t_{*}}^{T}\left(\frac{T}{\tau}\right)^{2 H-1}(T-\tau)^{2 \alpha+2 H-3} E_{\alpha, \alpha}^{2}\left(-\lambda_{k}(T-\tau)^{\alpha}\right) d \tau . \\
\lesssim & \int_{T-t_{*}}^{T}\left(\frac{T}{\tau}\right)^{2 H-1}(T-\tau)^{2 \alpha+2 H-3} d \tau .
\end{aligned}
$$

Clearly, this singular integral needs the condition $\alpha+H>1$ to guarantee the convergence. By the mean value theorem for the definite integral, there exists $T-t_{*}<\xi<T$ such that

$$
\begin{aligned}
& \int_{T-t_{*}}^{T}\left(\frac{T}{\tau}\right)^{2 H-1}(T-\tau)^{2 \alpha+2 H-3} d \tau \\
= & \left(\frac{T}{\xi}\right)^{2 H-1} \int_{T-t_{*}}^{T}(T-\tau)^{2 \alpha+2 H-3} d \tau \\
= & \left(\frac{T}{\xi}\right)^{2 H-1} \frac{t_{*}^{2 \alpha+2 H-2}}{2 \alpha+2 H-2} \lesssim t_{*}^{2 \alpha+2 H-2} .
\end{aligned}
$$

Combining the above estimate leads to

$$
I_{1}(T) \lesssim \frac{1}{\lambda_{k}^{2}} T^{2 H-2}+t_{*}^{2 \alpha+2 H-2}
$$


The estimate of $I_{2}(T)$. Using Lemma 2.2 and (4.4), we have

$$
\begin{aligned}
I_{2}(T)= & \int_{0}^{T} \tau^{1-2 H}\left[\left(\int_{\tau}^{T} u^{H-\frac{3}{2}}(u-\tau)^{H-\frac{1}{2}} d u\right)(T-\tau)^{\alpha-1} E_{\alpha, \alpha}\left(-\lambda_{k}(T-\tau)^{\alpha}\right)\right]^{2} d \tau \\
\lesssim & \int_{0}^{T} \tau^{1-2 H}\left(\int_{\tau}^{T} u^{H-\frac{3}{2}}(u-\tau)^{H-\frac{1}{2}} d u\right)^{2}(T-\tau)^{2 \alpha-2}\left(\frac{1}{1+\lambda_{k}(T-\tau)^{\alpha}}\right)^{2} d \tau \\
= & \int_{0}^{T-t_{*}} \tau^{1-2 H}\left(\int_{\tau}^{T-t_{*}} u^{H-\frac{3}{2}}(u-\tau)^{H-\frac{1}{2}} d u\right)^{2}(T-\tau)^{2 \alpha-2}\left(\frac{1}{1+\lambda_{k}(T-\tau)^{\alpha}}\right)^{2} d \tau \\
& +\int_{0}^{T-t_{*}} \tau^{1-2 H}\left(\int_{T-t_{*}}^{T} u^{H-\frac{3}{2}}(u-\tau)^{H-\frac{1}{2}} d u\right)^{2}(T-\tau)^{2 \alpha-2}\left(\frac{1}{1+\lambda_{k}(T-\tau)^{\alpha}}\right)^{2} d \tau \\
& +\int_{T-t_{*}}^{T} \tau^{1-2 H}\left(\int_{\tau}^{T} u^{H-\frac{3}{2}}(u-\tau)^{H-\frac{1}{2}} d u\right)^{2}(T-\tau)^{2 \alpha-2}\left(\frac{1}{1+\lambda_{k}(T-\tau)^{\alpha}} d \tau\right. \\
\lesssim & \int_{0}^{T-t_{*}} \tau^{1-2 H}\left(\int_{\tau}^{T-t_{*}} u^{H-\frac{3}{2}}(u-\tau)^{H-\frac{1}{2}} d u\right)^{2}(T-\tau)^{2 \alpha-2} \frac{1}{\lambda_{k}^{2}(T-\tau)^{2 \alpha}} d \tau \\
& +\int_{0}^{T-t_{*}} \tau^{1-2 H}\left(\int_{T-t_{*}}^{T} u^{H-\frac{3}{2}}(u-\tau)^{H-\frac{1}{2}} d u\right)^{2}(T-\tau)^{2 \alpha-2} \frac{1}{\lambda_{k}^{2}(T-\tau)^{2 \alpha}} d \tau \\
& +\int_{T-t_{*}}^{T} \tau^{1-2 H}\left(\int_{\tau}^{T} u^{H-\frac{3}{2}}(u-\tau)^{H-\frac{1}{2}} d u\right)^{2}(T-\tau)^{2 \alpha-2} d \tau \\
= & : J_{1}(T)+J_{2}(T)+J_{3}(T) .
\end{aligned}
$$

Next we estimate $J_{j}(T), j=1,2,3$, respectively.

For the term $J_{1}(T)$, we get

$$
\begin{aligned}
J_{1}(T)= & \frac{1}{\lambda_{k}^{2}} \int_{0}^{T-t_{*}} \tau^{1-2 H}(T-\tau)^{-2}\left(\int_{\tau}^{T-t_{*}} u^{H-\frac{3}{2}}(u-\tau)^{H-\frac{1}{2}} d u\right)^{2} d \tau \\
= & \frac{1}{\lambda_{k}^{2}} \int_{0}^{T-t_{*}} \tau^{1-2 H}(T-\tau)^{-2}\left[\int_{\tau}^{T-t_{*}} u^{2 H-2} \sum_{n=0}^{\infty}\left(\begin{array}{c}
H-\frac{1}{2} \\
n
\end{array}\right)(-1)^{n} u^{-n} \tau^{n} d u\right]^{2} d \tau \\
= & \frac{1}{\lambda_{k}^{2}} \int_{0}^{T-t_{*}} \tau^{1-2 H}(T-\tau)^{-2}\left[\sum_{n=0}^{\infty}\left(\begin{array}{c}
H-\frac{1}{2} \\
n
\end{array}\right)(-1)^{n} \tau^{n} \int_{\tau}^{T-t_{*}} u^{2 H-2-n} d u\right]^{2} d \tau \\
= & \frac{1}{\lambda_{k}^{2}} \int_{0}^{T-t_{*}} \tau^{1-2 H}(T-\tau)^{-2}\left[\left(T-t_{*}\right)^{2 H-1} \sum_{n=0}^{\infty}\left(\begin{array}{c}
H-\frac{1}{2} \\
n
\end{array}\right) \frac{(-1)^{n}}{2 H-1-n}\left(\frac{\tau}{T-t_{*}}\right)^{n}\right. \\
& \left.-\tau^{2 H-1} \sum_{n=0}^{\infty}\left(\begin{array}{c}
H-\frac{1}{2} \\
n
\end{array}\right) \frac{(-1)^{n}}{2 H-1-n}\right]^{2} d \tau \\
\leq & \frac{1}{\lambda_{k}^{2}} \int_{0}^{T-t_{*}} \tau^{1-2 H}(T-\tau)^{-2}\left[\left(T-t_{*}\right)^{4 H-2}+\tau^{4 H-2}\right] d \tau \\
= & \frac{1}{\lambda_{k}^{2}}\left(\left(T-t_{*}\right)^{4 H-2} \int_{0}^{T-t_{*}} \tau^{1-2 H}(T-\tau)^{-2} d \tau+\int_{0}^{T-t_{*}} \tau^{2 H-1}(T-\tau)^{-2} d \tau\right),
\end{aligned}
$$


where the condition $0<H<\frac{1}{2}$ is used to make the above singular integrals convergent. Hence

$$
\begin{aligned}
& J_{1}(T) \lesssim \frac{1}{\lambda_{k}^{2}}\left(\left(T-t_{*}\right)^{4 H-2} \int_{0}^{T-t_{*}} \tau^{1-2 H}(T-\tau)^{-2} d \tau+\int_{0}^{T-t_{*}} \tau^{2 H-1}(T-\tau)^{-2} d \tau\right) \\
& =\frac{1}{\lambda_{k}^{2}}\left[\left(T-t_{*}\right)^{4 H-2} T^{-2} \int_{0}^{T-t_{*}} \tau^{1-2 H}\left(\sum_{n=0}^{\infty}\left(\begin{array}{c}
-2 \\
n
\end{array}\right)(-1)^{n} T^{-n} \tau^{n}\right) d \tau\right. \\
& \left.+T^{-2} \int_{0}^{T-t_{*}} \tau^{2 H-1}\left(\sum_{n=0}^{\infty}\left(\begin{array}{c}
-2 \\
n
\end{array}\right)(-1)^{n} T^{-n} \tau^{n}\right) d \tau\right] \\
& =\frac{1}{\lambda_{k}^{2}}\left[T^{-2}\left(T-t_{*}\right)^{2 H} \sum_{n=0}^{\infty}\left(\begin{array}{c}
-2 \\
n
\end{array}\right) \frac{(-1)^{n}}{2-2 H+n}\left(\frac{T-t_{*}}{T}\right)^{n}\right. \\
& \left.+T^{-2}\left(T-t_{*}\right)^{2 H} \sum_{n=0}^{\infty}\left(\begin{array}{c}
-2 \\
n
\end{array}\right) \frac{(-1)^{n}}{2 H+n}\left(\frac{T-t_{*}}{T}\right)^{n}\right] \lesssim \frac{1}{\lambda_{k}^{2}} T^{2 H-2} .
\end{aligned}
$$

For the second term $J_{2}(T)$ in (4.6), we have

$$
\begin{aligned}
J_{2}(T)= & \frac{1}{\lambda_{k}^{2}} \int_{0}^{T-t_{*}} \tau^{1-2 H}(T-\tau)^{-2}\left(\int_{T-t_{*}}^{T} u^{H-\frac{3}{2}}(u-\tau)^{H-\frac{1}{2}} d u\right)^{2} d \tau \\
= & \frac{1}{\lambda_{k}^{2}} \int_{0}^{T-t_{*}} \tau^{1-2 H}(T-\tau)^{-2}\left[\sum_{n=0}^{\infty}\left(\begin{array}{c}
H-\frac{1}{2} \\
n
\end{array}\right)(-1)^{n} \tau^{n} \int_{T-t_{*}}^{T} u^{2 H-2-n} d u\right]^{2} d \tau \\
= & \frac{1}{\lambda_{k}^{2}} \int_{0}^{T-t_{*}} \tau^{1-2 H}(T-\tau)^{-2}\left[T^{2 H-1} \sum_{n=0}^{\infty}\left(\begin{array}{c}
H-\frac{1}{2} \\
n
\end{array}\right) \frac{(-1)^{n}}{2 H-1-n}\left(\frac{\tau}{T}\right)^{n}\right. \\
& \left.-\left(T-t_{*}\right)^{2 H-1} \sum_{n=0}^{\infty}\left(\begin{array}{c}
H-\frac{1}{2} \\
n
\end{array}\right) \frac{(-1)^{n}}{2 H-1-n}\left(\frac{\tau}{T-t_{*}}\right)^{n}\right]^{2} d \tau \\
\lesssim & \frac{1}{\lambda_{k}^{2}} \int_{0}^{T-t_{*}} \tau^{1-2 H}(T-\tau)^{-2}\left[T^{4 H-2}+\left(T-t_{*}\right)^{4 H-2}\right] d \tau \\
= & \frac{1}{\lambda_{k}^{2}}\left[T^{4 H-2}+\left(T-t_{*}\right)^{4 H-2}\right] T^{-2}\left(T-t_{*}\right)^{2-2 H} \sum_{n=0}^{\infty}\left(\begin{array}{c}
-2 \\
n
\end{array}\right) \frac{(-1)^{n}}{2-2 H+n}\left(\frac{T-t_{*}}{T}\right)^{n} \\
\lesssim & \frac{1}{\lambda_{k}^{2}} T^{2 H-2} .
\end{aligned}
$$

In (4.9), we have used the same tricks as those in (4.7) and (4.8). 
For the third term $J_{3}(T)$, we obtain

$$
\begin{aligned}
& J_{3}(T)=\int_{T-t_{*}}^{T} \tau^{1-2 H}(T-\tau)^{2 \alpha-2}\left(\int_{\tau}^{T} u^{H-\frac{3}{2}}(u-\tau)^{H-\frac{1}{2}} d u\right)^{2} d \tau \\
& =\int_{T-t_{*}}^{T} \tau^{1-2 H}(T-\tau)^{2 \alpha-2}\left(\sum_{n=0}^{\infty}\left(\begin{array}{c}
H-\frac{1}{2} \\
n
\end{array}\right)(-1)^{n} \tau^{n} \int_{\tau}^{T} u^{2 H-2-n} d u\right)^{2} d \tau \\
& =\int_{T-t_{*}}^{T} \tau^{1-2 H}(T-\tau)^{2 \alpha-2}\left(T^{2 H-1} \sum_{n=0}^{\infty}\left(\begin{array}{c}
H-\frac{1}{2} \\
n
\end{array}\right) \frac{(-1)^{n}}{2 H-1-n}\left(\frac{\tau}{T}\right)^{n}\right. \\
& \left.-\tau^{2 H-1} \sum_{n=0}^{\infty}\left(\begin{array}{c}
H-\frac{1}{2} \\
n
\end{array}\right) \frac{(-1)^{n}}{2 H-1-n}\right)^{2} d \tau \\
& \lesssim \int_{T-t_{*}}^{T} \tau^{1-2 H}(T-\tau)^{2 \alpha-2}\left(T^{4 H-2}+\tau^{4 H-2}\right) d \tau \\
& =T^{4 H+2 \alpha-4} \int_{T-t_{*}}^{T} \tau^{1-2 H}\left(\sum_{n=0}^{\infty}\left(\begin{array}{c}
2 \alpha-2 \\
n
\end{array}\right)(-1)^{n} T^{-n} \tau^{n}\right) d \tau \\
& +T^{2 \alpha-2} \int_{T-t_{*}}^{T} \tau^{2 H-1}\left(\sum_{n=0}^{\infty}\left(\begin{array}{c}
2 \alpha-2 \\
n
\end{array}\right)(-1)^{n} T^{-n} \tau^{n}\right) d \tau \\
& =T^{4 H+2 \alpha-4} \sum_{n=0}^{\infty}\left(\begin{array}{c}
2 \alpha-2 \\
n
\end{array}\right)(-1)^{n} T^{-n} \frac{T^{2-2 H+n}-\left(T-t_{*}\right)^{2-2 H+n}}{2-2 H+n} \\
& +T^{2 \alpha-2} \sum_{n=0}^{\infty}\left(\begin{array}{c}
2 \alpha-2 \\
n
\end{array}\right)(-1)^{n} T^{-n} \frac{T^{2 H+n}-\left(T-t_{*}\right)^{2 H+n}}{2 H+n} .
\end{aligned}
$$

Noting the range of $H$, we can use the differential mean value theorem and the Hölder continuity of $x^{2 H}$ to obtain

$$
J_{2}(T) \lesssim T^{2 H+2 \alpha-3} t_{*}+T^{2 \alpha-2}\left(t_{*}^{2 H} \vee t_{*}\left(T-t_{*}\right)^{2 H-1}\right) .
$$

Combining (4.6), (4.8), (4.9), and (4.10), we have

$$
I_{2}(T) \lesssim \lambda_{k}^{-2}+\left(t_{*}^{2 H} \vee t_{*}\right) .
$$

The estimate of $I_{3}(T)$. According to Lemma 2.5,

$$
\begin{aligned}
I_{3}(T) \lesssim & \int_{0}^{T}\left[\int_{\tau}^{T}\left[\int_{T-u}^{T-\tau} \frac{r^{\alpha-2}}{1+\lambda_{k} r^{\alpha}} d r\right]\left(\frac{u}{\tau}\right)^{H-\frac{1}{2}}(u-\tau)^{H-\frac{3}{2}} d u\right]^{2} d \tau \\
\lesssim & \int_{0}^{T-t_{*}}\left[\int_{\tau}^{T-t_{*}}\left[\int_{T-u}^{T-\tau} \frac{r^{\alpha-2}}{1+\lambda_{k} r^{\alpha}} d r\right]\left(\frac{u}{\tau}\right)^{H-\frac{1}{2}}(u-\tau)^{H-\frac{3}{2}} d u\right]^{2} d \tau \\
& +\int_{0}^{T-t_{*}}\left[\int_{T-t_{*}}^{T}\left[\int_{T-u}^{T-\tau} \frac{r^{\alpha-2}}{1+\lambda_{k} r^{\alpha}} d r\right]\left(\frac{u}{\tau}\right)^{H-\frac{1}{2}}(u-\tau)^{H-\frac{3}{2}} d u\right]^{2} d \tau \\
& +\int_{T-t_{*}}^{T}\left[\int_{\tau}^{T}\left[\int_{T-u}^{T-\tau} \frac{r^{\alpha-2}}{1+\lambda_{k} r^{\alpha}} d r\right]\left(\frac{u}{\tau}\right)^{H-\frac{1}{2}}(u-\tau)^{H-\frac{3}{2}} d u\right]^{2} d \tau \\
= & : K_{1}(T)+K_{2}(T)+K_{3}(T) .
\end{aligned}
$$

Next is to estimate $K_{j}(T), j=1,2,3$. 
For $K_{1}(T)$, a simple calculation gives

$$
\begin{aligned}
K_{1}(T) & \lesssim \int_{0}^{T-t_{*}}\left[\int_{\tau}^{T-t_{*}}\left[\frac{1}{\lambda_{k}} \int_{T-u}^{T-\tau} r^{-2} d r\right]\left(\frac{u}{\tau}\right)^{H-\frac{1}{2}}(u-\tau)^{H-\frac{3}{2}} d u\right]^{2} d \tau \\
& \lesssim \frac{1}{\lambda_{k}^{2}} \int_{0}^{T-t_{*}}\left[\int_{\tau}^{T-t_{*}}(T-u)^{-2}(u-\tau)^{H-\frac{1}{2}}\left(\frac{u}{\tau}\right)^{H-\frac{1}{2}} d u\right]^{2} d \tau
\end{aligned}
$$

Noting $H \in\left(0, \frac{1}{2}\right)$, we have

$$
\begin{aligned}
K_{1}(T) & \lesssim \frac{1}{\lambda_{k}^{2}} \int_{0}^{T-t_{*}}\left[\int_{0}^{T-t_{*}-\tau}(T-\tau-r)^{-2} r^{H-\frac{1}{2}} d r\right]^{2} d \tau \\
& \lesssim \frac{1}{\lambda_{k}^{2}} \int_{0}^{T-t_{*}}(T-\tau)^{-4}\left[\sum_{n=0}^{\infty}\left(\begin{array}{c}
-2 \\
n
\end{array}\right)(-1)^{n}(T-\tau)^{-n} \int_{0}^{T-t_{*}-\tau} r^{n+H-\frac{1}{2}} d r\right]^{2} d \tau \\
& =\frac{1}{\lambda_{k}^{2}} \int_{0}^{T-t_{*}}(T-\tau)^{-4}\left(T-t_{*}-\tau\right)^{2 H+1} d \tau\left[\sum_{n=0}^{\infty}\left(\begin{array}{c}
-2 \\
n
\end{array}\right)(-1)^{n} \frac{\left(T-t_{*}-\tau\right)^{n}}{(T-\tau)^{n}} \frac{1}{n+H+\frac{1}{2}}\right]^{2} \\
& \lesssim \frac{1}{\lambda_{k}^{2}} \int_{0}^{T-t_{*}}(T-\tau)^{2 H-3} d \tau \lesssim \frac{1}{\lambda_{k}^{2} t_{*}^{2-2 H}} .
\end{aligned}
$$

For $K_{2}(T)$, noting that $t_{*}<T-\tau<T$ and $0<T-u<t_{*}$ since $0<\tau<T-t_{*}$ and $T-t_{*}<u<T$, we get

$$
\begin{aligned}
\int_{T-u}^{T-\tau} \frac{r^{\alpha-2}}{1+\lambda_{k} r^{\alpha}} d r & \leq \int_{T-u}^{t_{*}} r^{\alpha-2} d r+\int_{t_{*}}^{T-\tau} \frac{1}{\lambda_{k}} r^{-2} d r \\
& \leq\left(t_{*}^{\alpha} \vee \frac{1}{\lambda_{k}}\right) \int_{T-u}^{T-\tau} r^{-2} d r \\
& \leq\left(t_{*}^{\alpha} \vee \frac{1}{\lambda_{k}}\right)(T-u)^{-2}(u-\tau) .
\end{aligned}
$$

As a result, we have for $H \in\left(0, \frac{1}{2}\right)$ that

$$
\begin{aligned}
K_{2}(T) & \lesssim \int_{0}^{T-t_{*}}\left[\int_{T-t_{*}}^{T}\left(t_{*}^{\alpha} \vee \frac{1}{\lambda_{k}}\right)(T-u)^{-2}(u-\tau)^{H-\frac{1}{2}}\left(\frac{u}{\tau}\right)^{H-\frac{1}{2}} d u\right]^{2} d \tau \\
& \lesssim\left(t_{*}^{2 \alpha} \vee \frac{1}{\lambda_{k}^{2}}\right) \int_{0}^{T-t_{*}}\left[\int_{T-t_{*}-\tau}^{T-\tau}(T-\tau-r)^{-2} r^{H-\frac{1}{2}} d r\right]^{2} d \tau \\
& \lesssim\left(t_{*}^{2 \alpha} \vee \frac{1}{\lambda_{k}^{2}}\right) \int_{0}^{T-t_{*}}(T-\tau)^{-4}\left[\sum_{n=0}^{\infty}\left(\begin{array}{c}
-2 \\
n
\end{array}\right)(-1)^{n}(T-\tau)^{-n} \int_{T-t_{*}-\tau}^{T-\tau} r^{n+H-\frac{1}{2}} d r\right]^{2} d \tau \\
& \lesssim\left(t_{*}^{2 \alpha} \vee \frac{1}{\lambda_{k}^{2}}\right) \int_{0}^{T-t_{*}}(T-\tau)^{2 H-3} d \tau \\
& \lesssim\left(t_{*}^{2 \alpha+2 H-2} \vee \frac{1}{\lambda_{k}^{2} t_{*}^{2-2 H}}\right) .
\end{aligned}
$$


For $K_{3}(T)$, since $0<T-u<T-\tau<t_{*}$ for $T-t_{*}<\tau<u<T$,

$$
\begin{aligned}
K_{3}(T) & \lesssim \int_{T-t_{*}}^{T}\left[\int_{\tau}^{T}\left[\int_{T-u}^{T-\tau} r^{\alpha-2} d r\right]\left(\frac{u}{\tau}\right)^{H-\frac{1}{2}}(u-\tau)^{H-\frac{3}{2}} d u\right]^{2} d \tau \\
& \lesssim \int_{T-t_{*}}^{T}\left[\int_{\tau}^{T}(T-u)^{\alpha-2}(u-\tau)^{H-\frac{1}{2}}\left(\frac{u}{\tau}\right)^{H-\frac{1}{2}} d u\right]^{2} d \tau \\
& \lesssim \int_{T-t_{*}}^{T}\left[\int_{0}^{T-\tau}(T-\tau-r)^{\alpha-2} r^{H-\frac{1}{2}} d r\right]^{2} d \tau \\
& \lesssim \int_{T-t_{*}}^{T}(T-\tau)^{2 \alpha+2 H-3} d \tau \lesssim t_{*}^{2 \alpha+2 H-2},
\end{aligned}
$$

where we have used the condition $\alpha+H>1$ again.

Combining the above estimates, we conclude that

$$
I_{3}(T) \lesssim\left(t_{*}^{2 \alpha+2 H-2} \vee \frac{1}{\lambda_{k}^{2} t_{*}^{2-2 H}}\right) .
$$

Finally, it follows from (4.5)-(4.12) that we obtain

$$
\begin{aligned}
& \mathbb{E}\left|\int_{0}^{T}(T-\tau)^{\alpha-1} E_{\alpha, \alpha}\left(-\lambda_{k}(T-\tau)^{\alpha}\right) d B^{H}(\tau)\right|^{2} \\
\lesssim & \max \left\{t_{*}^{2 \alpha+2 H-2}, \lambda_{k}^{-2} t_{*}^{2-2 H}, \lambda_{k}^{-2}, t_{*}^{2 H}, t_{*}\right\} .
\end{aligned}
$$

4.2. The case $H \in\left(\frac{1}{2}, 1\right)$. Set $\tilde{p}=T-p, \tilde{q}=T-q$. From (4.2) and (A.4), we have

$$
\begin{aligned}
& \mathbb{E}\left|\int_{0}^{T}(T-\tau)^{\alpha-1} E_{\alpha, \alpha}\left(-\lambda_{k}(T-\tau)^{\alpha}\right) d B^{H}(\tau)\right|^{2} \\
= & \alpha_{H} \int_{0}^{T} \int_{0}^{T}(T-p)^{\alpha-1} E_{\alpha, \alpha}\left(-\lambda_{k}(T-p)^{\alpha}\right)(T-q)^{\alpha-1} E_{\alpha, \alpha}\left(-\lambda_{k}(T-q)^{\alpha}\right)|p-q|^{2 H-2} d p d q \\
= & \alpha_{H} \int_{0}^{T} \int_{0}^{T} \tilde{p}^{\alpha-1} E_{\alpha, \alpha}\left(-\lambda_{k} \tilde{p}^{\alpha}\right) \tilde{q}^{\alpha-1} E_{\alpha, \alpha}\left(-\lambda_{k} \tilde{q}^{\alpha}\right)|\tilde{q}-\tilde{p}|^{2 H-2} d \tilde{p} d \tilde{q} \\
= & \alpha_{H}\left(\int_{0}^{t_{*}} \int_{0}^{t_{*}}+\int_{t_{*}}^{T} \int_{t_{*}}^{T}+\int_{0}^{t_{*}} \int_{t_{*}}^{T}+\int_{t_{*}}^{T} \int_{0}^{t_{*}}\right) \tilde{p}^{\alpha-1} E_{\alpha, \alpha}\left(-\lambda_{k} \tilde{p}^{\alpha}\right) \tilde{q}^{\alpha-1} E_{\alpha, \alpha}\left(-\lambda_{k} \tilde{q}^{\alpha}\right)|\tilde{q}-\tilde{p}|^{2 H-2} d \tilde{p} d \tilde{q} \\
= & : \alpha_{H}\left(M_{1}(T)+M_{2}(T)+M_{3}(T)+M_{4}(T)\right) .
\end{aligned}
$$

We choose $t_{*}$ as that in (4.4). It is easy to see that $M_{3}(T)=M_{4}(T)$. Then we only need to discuss $M_{j}(T), j=1,2,3$.

For the term $M_{1}(T)$, we can use the same analysis in Subsection 3.2 to get

$$
\begin{aligned}
M_{1}(T) & =\int_{0}^{t_{*}} \int_{0}^{t_{*}} \tilde{p}^{\alpha-1} E_{\alpha, \alpha}\left(-\lambda_{k} \tilde{p}^{\alpha}\right) \tilde{q}^{\alpha-1} E_{\alpha, \alpha}\left(-\lambda_{k} \tilde{q}^{\alpha}\right)|\tilde{q}-\tilde{p}|^{2 H-2} d \tilde{p} d \tilde{q} \\
& \lesssim \int_{0}^{t_{*}} \int_{0}^{t_{*}} \tilde{p}^{\alpha-1} \tilde{q}^{\alpha-1}|\tilde{q}-\tilde{p}|^{2 H-2} d \tilde{p} d \tilde{q} \quad(\text { noting } \alpha>0) \\
& \lesssim t_{*}^{2 \alpha+2 H-2} .
\end{aligned}
$$


For the term $M_{2}(T)$, it is easy to verify that

$$
\begin{aligned}
M_{2}(T) & =\int_{t_{*}}^{T} \int_{t_{*}}^{T} \tilde{p}^{\alpha-1} E_{\alpha, \alpha}\left(-\lambda_{k} \tilde{p}^{\alpha}\right) \tilde{q}^{\alpha-1} E_{\alpha, \alpha}\left(-\lambda_{k} \tilde{q}^{\alpha}\right)|\tilde{q}-\tilde{p}|^{2 H-2} d \tilde{p} d \tilde{q} \\
& \lesssim \frac{1}{\lambda_{k}^{2}} \int_{t_{*}}^{T} \int_{t_{*}}^{T} \frac{1}{\tilde{p}} \frac{1}{\tilde{q}}|\tilde{q}-\tilde{p}|^{2 H-2} d \tilde{p} d \tilde{q} \\
& \leq \frac{1}{\lambda_{k}^{2} t_{*}^{2}} \int_{t_{*}}^{T} \int_{t_{*}}^{T}|\tilde{q}-\tilde{p}|^{2 H-2} d \tilde{p} d \tilde{q} \\
& =\frac{1}{\lambda_{k}^{2} t_{*}^{2}}\left(\int_{t_{*}}^{T} \int_{t_{*}}^{\tilde{q}}(\tilde{q}-\tilde{p})^{2 H-2} d \tilde{p} d \tilde{q}+\int_{t_{*}}^{T} \int_{\tilde{q}}^{T}(\tilde{p}-\tilde{q})^{2 H-2} d \tilde{p} d \tilde{q}\right) \\
& =\frac{2}{\lambda_{k}^{2} t_{*}^{2}} \int_{t_{*}}^{T} \int_{t_{*}}^{\tilde{q}}(\tilde{q}-\tilde{p})^{2 H-2} d \tilde{p} d \tilde{q} \quad\left(\operatorname{noting} H>\frac{1}{2}\right) \\
& =\frac{2}{\lambda_{k}^{2} t_{*}^{2}} \int_{t_{*}}^{T} \frac{\left(\tilde{q}-t_{*}\right)^{2 H-1}}{2 H-1} d \tilde{q} \\
& =\frac{2}{\lambda_{k}^{2} t_{*}^{2}} \frac{\left(T-t_{*}\right)^{2 H}}{2 H(2 H-1)} \lesssim \frac{1}{\lambda_{k}^{2} t_{*}^{2}} .
\end{aligned}
$$

For the term $M_{3}(T)$, we may similarly have

$$
\begin{aligned}
M_{3}(T) & =\int_{0}^{t_{*}} \int_{t_{*}}^{T} \tilde{p}^{\alpha-1} E_{\alpha, \alpha}\left(-\lambda_{k} \tilde{p}^{\alpha}\right) \tilde{q}^{\alpha-1} E_{\alpha, \alpha}\left(-\lambda_{k} \tilde{q}^{\alpha}\right)|\tilde{q}-\tilde{p}|^{2 H-2} d \tilde{p} d \tilde{q} \\
& \lesssim \frac{1}{\lambda_{k}} \int_{0}^{t_{*}} \int_{t_{*}}^{T} \frac{1}{\tilde{p}} \tilde{q}^{\alpha-1}(\tilde{p}-\tilde{q})^{2 H-2} d \tilde{p} d \tilde{q} \\
& \leq \frac{1}{\lambda_{k} t_{*}} \int_{0}^{t_{*}} \int_{t_{*}}^{T} \tilde{q}^{\alpha-1}(\tilde{p}-\tilde{q})^{2 H-2} d \tilde{p} d \tilde{q} \\
& \left.=\frac{1}{\lambda_{k} t_{*}} \int_{0}^{t_{*}} \tilde{q}^{\alpha-1} \frac{(T-\tilde{q})^{2 H-1}-\left(t_{*}-\tilde{q}\right)^{2 H-1}}{2 H-1} d \tilde{q} \quad \text { (noting } H>\frac{1}{2}\right) \\
& \lesssim \frac{1}{\lambda_{k} t_{*}} \int_{0}^{t_{*}} \tilde{q}^{\alpha-1}(T-\tilde{q})^{2 H-1} d \tilde{q} \\
& \lesssim \frac{1}{\lambda_{k} t_{*}} \int_{0}^{t_{*}} \tilde{q}^{\alpha-1} d \tilde{q} \lesssim \frac{1}{\lambda_{k}} t_{*}^{\alpha-1} .
\end{aligned}
$$

It follows from (4.14)-(4.17) that we obtain the estimate

$$
\mathbb{E}\left|\int_{0}^{T}(T-\tau)^{\alpha-1} E_{\alpha, \alpha}\left(-\lambda_{k}(T-\tau)^{\alpha}\right) d B^{H}(\tau)\right|^{2} \lesssim \max \left\{t_{*}^{2 \alpha+2 H-2}, \frac{1}{\lambda_{k}^{2} t_{*}^{2}}, \frac{1}{\lambda_{k}} t_{*}^{\alpha-1}\right\},
$$

which is crucial to explain the instability of the inverse problem.

4.3. Instability. Based on the analysis above, we can obtain the following theorem which shows that it is unstable to reconstruct $f$ and $|g|$.

Theorem 4.4. The problem of recovering the source terms $f$ and $|g|$ is unstable. Moreover, the following estimates hold

$$
\left|\int_{0}^{T}(T-\tau)^{\alpha-1} E_{\alpha, \alpha}\left(-\lambda_{k}(T-\tau)^{\alpha}\right) h(\tau) d \tau\right| \lesssim \lambda_{k}^{-1}
$$


and

$$
\mathbb{E}\left|\int_{0}^{T}(T-\tau)^{\alpha-1} E_{\alpha, \alpha}\left(-\lambda_{k}(T-\tau)^{\alpha}\right) d B^{H}(\tau)\right|^{2} \lesssim \lambda_{k}^{-\beta}
$$

where

$$
\beta=\left\{\begin{array}{ll}
\min \{2 \gamma(\alpha+H-1), 2-2 \gamma(H-1), 2 H, \gamma\}, & 0<H<\frac{1}{2}, \\
\min \{2 \gamma(\alpha+H-1), 2(1-\gamma), 1-\gamma(1-\alpha)\}, & \frac{1}{2}<H<1,
\end{array} \quad 0<\gamma<1, \alpha+H>1\right.
$$

and

$$
\beta=\min \{\gamma(2 \alpha-1), 1-\gamma\}, \quad H=\frac{1}{2}, 0<\gamma<1, \alpha>\frac{1}{2} .
$$

Proof. For (4.19), one can refer to [29, Lemma 4.4]. For (4.20), one can obtain it by choosing $t_{*}=\lambda_{k}^{-\gamma}, 0<\gamma<1$ in (4.13) and (4.18). Here the case $H=\frac{1}{2}$ can be seen in [29, Lemma 4.4]. For $\alpha=1$, one can use $e^{-x}<\frac{1}{1+x}, x \geq 0$ to obtain the same results. Since $\lambda_{k} \rightarrow \infty$ as $k \rightarrow \infty$, the instability follows easily from the estimates (4.19) -(4.20) and the reconstruction formulas (4.1)(4.2).

\section{Conclusion}

In this paper, we have studied an inverse random source problem for the time fractional diffusion equation driven by fractional Brownian motions. By the analysis, we deduce the relationship of the time fractional order $\alpha$ and the Hurst index $H$ in the fractional Brownian motion to ensure that the solution is well-defined for the stochastic time fractional diffusion equation. We show that the direct problem is well-posed when $\alpha+H>1$ and the inverse source problem has a unique solution. But the inverse problem is ill-posed in the sense that a small deviation of the data may lead to a huge error in the reconstruction.

There are a few related interesting observation. First, if the Laplacian operator is also fractional, the method can be directly applied and all the results can be similarly proved. Second, for $1<\alpha \leq 2$, the direct problem can be shown to be well-posed since Lemma 2.2 is still valid. However, the inverse problem may not have a unique solution. The reason is that Lemma 2.7 is not true any more for $1<\alpha \leq 2$. Finally, we mention that the numerics needs to be investigated. Clearly, some regularization techniques are indispensable in order to suppress the instability of the inverse problem. Another challenge is to how to compute the integrals efficiently and accurately. We will report the numerical results elsewhere in the future.

\section{Appendix A. Fractional Brownian motion}

In the appendix, we briefly introduce the fractional Brownian motion (fBm) and present some results which are used in this work.

A.1. Definition and Hölder continuity. A one dimensional fractional Brownian motion (fBm) $B^{H}$ with the Hurst parameter $H \in(0,1)$ is a centered Gaussian process (i.e., $B^{H}(0)=0$ ) determined by its covariance function

$$
R_{H}(t, s)=\mathbb{E}\left[B^{H}(t) B^{H}(s)\right]=\frac{1}{2}\left(t^{2 H}+s^{2 H}-|t-s|^{2 H}\right)
$$

for any $s, t \geq 0$. In particular, if $H=\frac{1}{2}, B^{H}$ turns to be the standard Brownian motion, which is usually denoted by $W$, with covariance function $R_{H}(t, s)=t \wedge s$.

The increments of fBms satisfies

$$
\mathbb{E}\left[\left(B^{H}(t)-B^{H}(s)\right)\left(B^{H}(s)-B^{H}(r)\right)\right]=\frac{1}{2}\left[(t-r)^{2 H}-(t-s)^{2 H}-(r-s)^{2 H}\right]
$$


and

$$
\mathbb{E}\left[\left(B^{H}(t)-B^{H}(s)\right)^{2}\right]=(t-s)^{2 H}
$$

for any $0<r<s<t$. It then indicates that the increments of $B^{H}$ in disjoint intervals are linearly dependent except for the case $H=\frac{1}{2}$, and the increments are stationary since its moment depends only on the length of the interval.

Based on the moment estimates and the Kolmogorov continuity criterion, it holds for any $\epsilon>0$ and $s, t \in[0, T]$ that

$$
\left|B^{H}(t)-B^{H}(s)\right| \leq C|t-s|^{H-\epsilon}
$$

almost surely with constant $C$ depending on $\epsilon$ and $T$. That is, $H$ represents the regularity of $B^{H}$ : the trajectories of fractional Brownian motion $B^{H}$ with Hurst parameter $H \in(0,1)$ are $(H-\epsilon)$-Hölder continuous.

A.2. Representation of $\mathbf{f B m}$ and integration. For a fractional Brownian motion $B^{H}$ with $H \in$ $(0,1)$, it has the following Wiener integral representation

$$
B^{H}(t)=\int_{0}^{t} K_{H}(t, s) d W(s)
$$

with $K_{H}$ being a square integrable kernel and $W$ being the standard Brownian motion (i.e., $H=\frac{1}{2}$ ).

For a fixed interval $[0, T]$, denote by $\mathcal{E}$ the space of step functions on $[0, T]$ and by $\mathcal{H}$ the closure of $\mathcal{E}$ with respect to the product

$$
\left\langle\mathbf{1}_{[0, t]}, \mathbf{1}_{[0, s]}\right\rangle_{\mathcal{H}}=R_{H}(t, s),
$$

where $\mathbf{1}_{[0, t]}, \mathbf{1}_{[0, s]}$ are the characteristic functions. Define the linear operator $K_{H, T}^{*}: \mathcal{E} \rightarrow L^{2}(0, T)$ by

$$
\left(K_{H, T}^{*} \psi\right)(s)=K_{H}(T, s) \psi(s)+\int_{s}^{T}(\psi(u)-\psi(s)) \frac{\partial K_{H}(u, s)}{\partial u} d u
$$

where

$$
\frac{\partial K_{H}(u, s)}{\partial u}=c_{H}\left(\frac{u}{s}\right)^{H-\frac{1}{2}}(u-s)^{H-\frac{3}{2}}
$$

and $c_{H}$ is a constant given below depending on $H$. Then $K_{H, T}^{*}$ is an isometry from $\mathcal{E}$ to $L^{2}(0, T)$ (see e.g. [30, 37]), and the integral with respect to $B^{H}$ can be defined for functions $\varphi$ satisfying

$$
\|\psi\|_{|\mathcal{H}|}^{2}:=\langle\psi, \psi\rangle_{\mathcal{H}}<\infty
$$

and (see e.g. [30, 37])

$$
\int_{0}^{t} \psi(s) d B^{H}(s)=\int_{0}^{T} \psi(s) \mathbf{1}_{[0, t]}(s) d B^{H}(s)=\int_{0}^{T}\left[K_{H, T}^{*}\left(\psi \mathbf{1}_{[0, t]}\right)\right](s) d W(s)
$$

for any $t \in[0, T]$. Hence, according to the Itô isometry,

$$
\mathbb{E}\left[\int_{0}^{t} \psi(s) d B^{H}(s) \int_{0}^{t} \phi(s) d B^{H}(s)\right]=\left\langle K_{H, T}^{*}\left(\psi \mathbf{1}_{[0, t]}\right), K_{H, T}^{*}\left(\phi \mathbf{1}_{[0, t]}\right)\right\rangle_{L^{2}(0, T)} .
$$

A.2.1. The case $H \in\left(\frac{1}{2}, 1\right)$. For the case $H \in\left(\frac{1}{2}, 1\right)$, the covariance function $R_{H}$ of $B^{H}$ satisfies

$$
\begin{aligned}
R_{H}(t, s) & =\alpha_{H} \int_{0}^{t} \int_{0}^{s}|r-u|^{2 H-2} d u d r \\
& =\alpha_{H} \int_{0}^{T} \int_{0}^{T} \mathbf{1}_{[0, t]}(r) \mathbf{1}_{[0, s]}(u)|r-u|^{2 H-2} d u d r
\end{aligned}
$$

with $\alpha_{H}=H(2 H-1)$. The square integrable kernel has form

$$
K_{H}(t, s)=c_{H} \int_{s}^{t}\left(\frac{u}{s}\right)^{H-\frac{1}{2}}(u-s)^{H-\frac{3}{2}} d u
$$


with $c_{H}=\left(\frac{\alpha_{H}}{\beta\left(2-2 H, H-\frac{1}{2}\right)}\right)^{\frac{1}{2}}$ such that

$$
\begin{aligned}
\left\langle\mathbf{1}_{[0, t]}, \mathbf{1}_{[0, s]}\right\rangle_{\mathcal{H}}=R_{H}(t, s) & =\alpha_{H} \int_{0}^{T} \int_{0}^{T} \mathbf{1}_{[0, t]}(r) \mathbf{1}_{[0, s]}(u)|r-u|^{2 H-2} d u d r \\
& =\int_{0}^{T} \mathbf{1}_{[0, t]}(u) \mathbf{1}_{[0, s]}(u) K_{H}(t, u) K_{H}(s, u) d u,
\end{aligned}
$$

and $K_{H, T}^{*}$ in (A.1) turns to be

$$
\left(K_{H, T}^{*} \psi\right)(s)=\int_{s}^{T} \psi(u) \frac{\partial K_{H}(u, s)}{\partial u} d u
$$

By noting that

$$
\left(K_{H, T}^{*} \mathbf{1}_{[0, t]}\right)(s)=\int_{s}^{T} \mathbf{1}_{[0, t]}(u) \frac{\partial K_{H}(u, s)}{\partial u} d u=\mathbf{1}_{[0, t]}(s) \int_{s}^{t} \frac{\partial K_{H}(u, s)}{\partial u} d u=\mathbf{1}_{[0, t]}(s) K_{H}(t, s),
$$

one get

$$
\begin{aligned}
\left\langle\mathbf{1}_{[0, t]}, \mathbf{1}_{[0, s]}\right\rangle_{\mathcal{H}} & =\int_{0}^{T} \mathbf{1}_{[0, t]}(u) \mathbf{1}_{[0, s]}(u) K_{H}(t, u) K_{H}(s, u) d u \\
& =\int_{0}^{T}\left(K_{H, T}^{*} \mathbf{1}_{[0, t]}\right)(u)\left(K_{H, T}^{*} \mathbf{1}_{[0, s]}\right)(u) d u \\
& =\left\langle K_{H, T}^{*} \mathbf{1}_{[0, t]}, K_{H, T}^{*} \mathbf{1}_{[0, s]}\right\rangle_{L^{2}(0, T)} .
\end{aligned}
$$

In this case, (A.2) can be calculated as follows

$$
\begin{aligned}
& \mathbb{E}\left[\int_{0}^{t} \psi(s) d B^{H}(s) \int_{0}^{t} \phi(s) d B^{H}(s)\right] \\
= & \left\langle K_{H, T}^{*}\left(\psi \mathbf{1}_{[0, t]}\right), K_{H, T}^{*}\left(\phi \mathbf{1}_{[0, t]}\right)\right\rangle_{L^{2}(0, T)} \\
= & \left\langle\psi \mathbf{1}_{[0, t]}, \phi \mathbf{1}_{[0, t]}\right\rangle_{\mathcal{H}} \\
= & \alpha_{H} \int_{0}^{t} \int_{0}^{t} \psi(r) \phi(u)|r-u|^{2 H-2} d u d r
\end{aligned}
$$

according to (A.3), which is used in (3.6).

A.2.2. The case $H \in\left(0, \frac{1}{2}\right)$. If the trajectories of $B^{H}$ is less regular than the case above with $H \in\left(0, \frac{1}{2}\right)$, the square integrable kernel $K_{H}$ has the following form instead

$$
K_{H}(t, s)=c_{H}\left[\left(\frac{t}{s}\right)^{H-\frac{1}{2}}(t-s)^{H-\frac{1}{2}}-\left(H-\frac{1}{2}\right) s^{\frac{1}{2}-H} \int_{s}^{t} u^{H-\frac{3}{2}}(u-s)^{H-\frac{1}{2}} d u\right]
$$

with $c_{H}=\left(\frac{2 H}{(1-2 H) \beta\left(1-2 H, H+\frac{1}{2}\right)}\right)^{\frac{1}{2}}$ such that

$$
R_{H}(t, s)=\int_{0}^{t \wedge s} K_{H}(t, u) K_{H}(s, u) d u
$$

similar to (A.3). Utilizing the fact (see [37])

$$
\left[K_{H, T}^{*}\left(\psi \mathbf{1}_{[0, t]}\right)\right](s)=\left[\left(K_{H, t}^{*} \psi\right)(s)\right] \mathbf{1}_{[0, t]}(s), \quad \forall t \in[0, T],
$$


where $K_{H, t}^{*}$ is defined in (A.1), we may rewrite (A.2) into

$$
\begin{aligned}
\mathbb{E}\left[\int_{0}^{t} \psi(s) d B^{H}(s) \int_{0}^{t} \phi(s) d B^{H}(s)\right] & =\left\langle K_{H, T}^{*}\left(\psi \mathbf{1}_{[0, t]}\right), K_{H, T}^{*}\left(\phi \mathbf{1}_{[0, t]}\right)\right\rangle_{L^{2}(0, T)} \\
& =\left\langle K_{H, t}^{*} \psi, K_{H, t}^{*} \phi\right\rangle_{L^{2}(0, t)},
\end{aligned}
$$

which is used in Subsection 3.2 and (4.14).

\section{REFERENCES}

[1] E.E. Adams and L.W. Gelhar, Field study of dispersion in a heterogeneous aquifer 2. Spatial moments analysis, Water Resources Res., 28 (1992), 3293-307.

[2] T.S. Aleroev, M. Kirane, and S.A. Malik, Determination of a source term for a time fractional diffusion equation with an integral type over-determining condition, Electronic Journal of Differential Equations, 270 (2013), 1-16.

[3] S. Aziz and S.A. Malik, Identification of an unknown source term for a time fractional fourth-order parabolic equation, Electronic Journal of Differential Equations, 293 (2016), 1-20, 2016.

[4] G. Bao, C. Chen, and P. Li, Inverse random source scattering problems in several dimensions, SIAM/ASA J. Uncertain. Quantif., 4 (2016), 1263-1287.

[5] G. Bao, C. Chen, and P. Li, Inverse random source scattering for elastic waves, SIAM Journal on Numerical Analysis, 55 (2017), 2616-2643.

[6] G. Bao, S.N. Chow, P. Li, and H. Zhou, Numerical solution of an inverse medium scattering problem with a stochastic source, Inverse Problems, 26 (2010), 074014.

[7] G. Bao, S.N. Chow, P. Li, and H. Zhou, An inverse random source problem for the Helmholtz equation, Math. Comp., 83 (2014), 215-233.

[8] A. Devaney, The inverse problem for random sources, Journal of Mathematical Physics, 20 (1979), $1687-1691$.

[9] K.M. Furati, O.S. Iyiola, and M. Kirane, An inverse problem for a generalized fractional diffusion, Applied Mathematics and Computations, 249 (2014), 24-31, 2014.

[10] M. Ginoa, S. Cerbelli, and H.E. Roman, Fractional diffusion equation and relaxation in complex viscoelastic materials, Phys. A, 191 (1992), 449-453.

[11] R. Gorenflo and F. Mainardi, Some recent advances in theory and simulation of fractional diffusion processes, J. Comput. Appl. Math., 229 (2009), 400-415.

[12] R. Goreno, A.A. Kilbas, F. Mainardi, and S.V. Rogosin, Mittag-Leffler Functions, Related Topics and Applications, Springer, Heidelberg, 2014.

[13] Y. Hatano and N. Hatano, Dispersive transport of ions in column experiments: an explanation of long-tailed profiles, Water Resources Research, 34 (1998), 1027-1034.

[14] I.M. Ismailov and M. Cicek, Inverse source problem for a time-fractional diffusion equation with nonlocal boundary conditions, Appl. Math. Model., 40 (2016), 4891-4899.

[15] J.X. Jia, J.G. Peng, and J.Q. Yang, Harnack's inequality for a space-time fractional diffusion equation and applications to an inverse source problem, Journal of Differential Equations, 262 (2017), 4415-4450.

[16] B.T. Jin and W. Rundell, A tutorial on inverse problems for anomalous diffusion processes, Inverse Problems, 31 (2015), 035003.

[17] A.A. Kilbas, H.M. Srivastava, and J.J. Trujillo, Theory and Applications of Fractional Differential Equations, volume 204 of North-Holland Mathematics Studies, Elsevier Science B.V., Amsterdam, 2006.

[18] M. Kirane and A.S. Malik, Determination of an unknown source term and the temperature distribution for the linear heat equation involving fractional derivative in time, Appl. Math. Comput., 218 (2011), 163-170.

[19] M. Kirane, A.S. Malik, M.A. Al-Gwaiz, An inverse source problem for a two dimensional time fractional diffusion equation with nonlocal boundary conditions, Math. Methods Appl. Sci., 36 (2013), 1056-1069.

[20] M. Li, C. Chen, and P. Li, Inverse random source scattering for the helmholtz equation in inhomogeneous media, Inverse Problems, 34 (2017), 015003.

[21] P. Li, An inverse random source scattering problem in inhomogeneous media, Inverse Problems, 27 (2011), 035004.

[22] P. Li and G. Yuan, Stability on the inverse random source scattering problem for the one-dimensional Helmholtz equation, Journal of Mathematical Analysis and Applications, 450 (2017), 872-887.

[23] Y.S. Li and T. Wei, An inverse time-dependent source problem for a time-space fractional diffusion equation, Applied Mathematics and Computation, 336 (2018), 257-271.

[24] Y.K. Liu and Z.D. Zhang, Reconstruction of the temporal component in the source term of a (time-fractional) diffusion equation, J. Phys. A: Math. Theor., 50 (2017), 305203.

[25] Y. Luchko, W. Rundell, M. Yamamoto, and L.H. Zuo, Uniqueness and reconstruction of an unknown semilinear term in a time-fractional reaction diffusion equation, Inverse Problems, 29 (2013) 065019. 
[26] R. Metzler and J. Klafter, The random walk's guide to anomalous diffusion: a fractional dynamics approach, Physics Reports, 339 (2000), 1-77.

[27] D.A. Murio and C.E. Mejía, Source terms identification for time fractional diffusion equation, Rev. Colomb. Mat., 42 (2008), 25-46.

[28] E. Di Nezza, G. Palatucci, and E. Valdinoci, Hitchhiker's guide to the fractional Sobolev spaces. Bull. Sci. math., 136 (2012), 521-573.

[29] P. Niu, T. Helin, and Z. Zhang, An inverse random source problem in a stochastic fractional diffusion equation, arXiv: 1810.03144

[30] D. Nualart, The Malliavin Calculus and Related Topics, Probability and its Applications (New York), SpringerVerlag, Berlin, second edition, 2006.

[31] I. Podlubny, Fractional Differential Equations, volume 198 of Mathematics in Science and Engineering, Academic Press, Inc., San Diego, CA, 1999.

[32] H. Pollard, The completely monotonic character of the Mittag-Leffler function $E_{a}(-x)$. Bull. Amer. Math. Soc., 54 (1948), 1115-1116.

[33] K. Sakamoto and M. Yamamoto, Initial value/boundary value problems for fractional diffusion-wave equations and applications to some inverse problems, J. Math. Anal. Appl., 382 (2011), 426-447.

[34] K. Sakamoto and M. Yamamoto, Inverse source problem with a final overdetermination for a fractional diffusion equation, Mathematical Control \& Related Fields, 1 (2011), 509-518.

[35] S. Tatar, R. Tinaztepe, and S. Ulusoy, Determination of an unknown source term in a space-time fractional diffusion equation, Journal of Fractional Calculus and Applications, 6 (2015), 83-90.

[36] S. Tatar and S. Ulusoy, An inverse source problem for a one-dimensional space-time fractional diffusion equation, Applicable Analysis, 94 (2015), 2233-2244.

[37] S. Tindel, C.A. Tudor, and F. Viens, Stochastic evolution equations with fractional Brownian motion, Probab. Theory Related Fields, 127 (2003), 186-204.

[38] N.H. Tuan and L.D. Long, Fourier truncation method for an inverse source problem for space-time fractional diffusion equation, Electron. J. Differ. Equ., 122 (2017).

[39] J.G. Wang, Y.B. Zhou, and T. Wei, Two regularization methods to identify a space-dependent source for the time-fractional diffusion equation, Applied Numerical Mathematics, 68 (2013), 39-57.

[40] W.Y. Wang, M. Yamamoto, and B. Han, Numerical method in reproducing kernel space for an inverse source problem for the fractional diffusion equation, Inverse Problems, 29 (2013), 095009.

[41] T. Wei, L. Sun, and Y. Li, Uniqueness for an inverse space-dependent source term in a multi-dimensional timefractional diffusion equation, Applied Mathematics Letters, 61 (2016), 108-113.

[42] T. Wei and J.G. Wang, A modified quasi-boundary value method for an inverse source problem of the timefractional diffusion equation, Applied Numerical Mathematics, 78 (2014), 95-111.

[43] T. Wei and Z. Zhang, Reconstruction of a time-dependent source term in a time-fractional diffusion equation, Eng. Anal. Bound. Elem., 37 (2013), 23-31.

[44] F.R. Xavier and R.O. Xavier, Boundary regularity for the fractional heat equation, RACSAM, 110 (2016), 49-64.

[45] M. Yamamoto and Y. Zhang, Conditional stability in determining a zeroth-order coefficient in a half-order fractional diffusion equation by a Carleman estimate, Inverse Problems, 28 (2012), 105010.

[46] F. Yang, X. Liu, X. Li, and C. Ma, Landweber iterative regularization method for identifying the unknown source of the time-fractional diffusion equation, Adv. Differ. Equa., 388 (2017).

[47] Y. Zhang and X. Xu, Inverse source problem for a fractional diffusion equation, Inverse Problems, 27 (2011), 035010 .

School of Mathematics and Statistics, Xidian University, Xi'An, 713200, P. R. China

E-mail address: xiaolifeng@xidian.edu.cn

Department of Mathematics, Purdue University, West Lafayette, Indiana 47907, USA

E-mail address: lipeijun@math.purdue.edu

Department of Mathematics, Purdue University, West Lafayette, Indiana 47907, USA

E-mail address: wang4191@purdue.edu 